VA

واقع إنتاج محصول القمح فى مصر فى ظل المتغيرات العالمية والسياسات السعرية التأثيرية

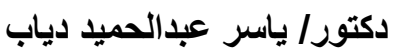

قسم الاقتصاد الزراعي - كلية الزراعة عبدابل دياب جامعة أسيوط

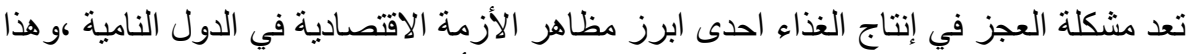

مقدمة:

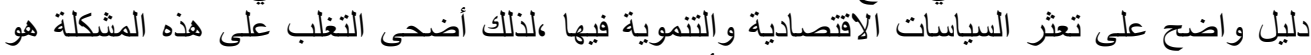

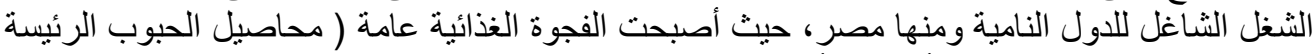
بشكل خاص) تتفاقم سنة بعد أخرى وأصبحت تلإلك المشكلة مزدوجة فهي ليست اقتصادية فحسب وإنة إنما مشكلة سياسية أيضا.

ولقد كان تأثير إصلاح السياسات الاقتصادية بما يتفق مع قوى السوق على حالة الأمن الغذائي

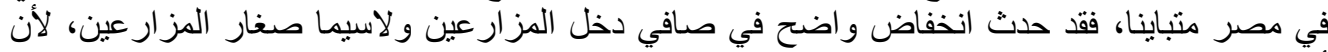

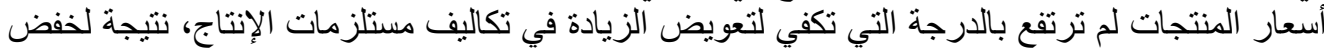

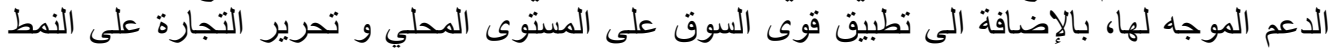

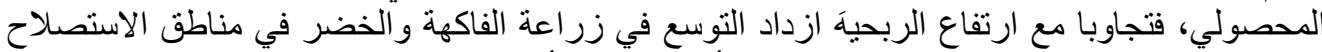

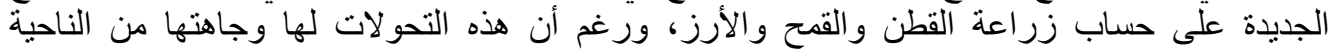

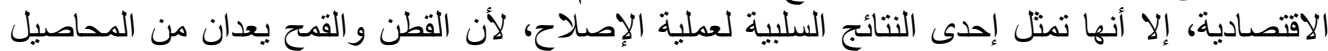

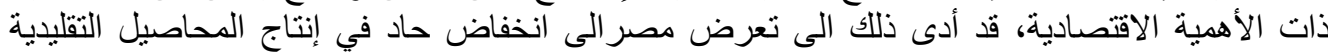

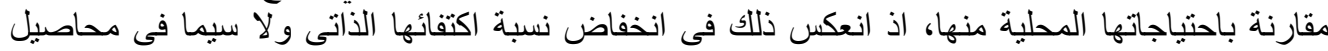

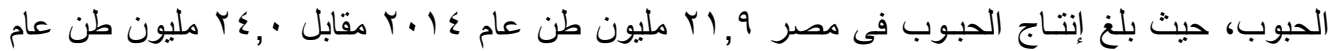

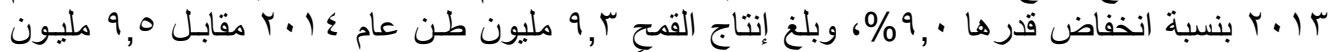

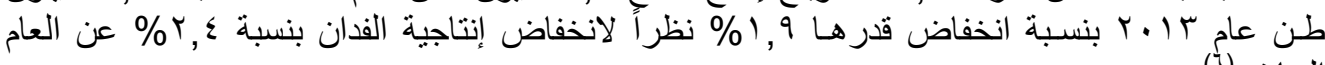
(السابق (י).

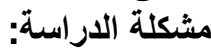

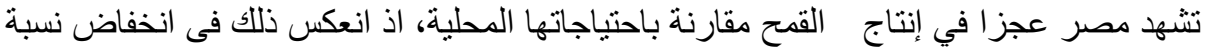

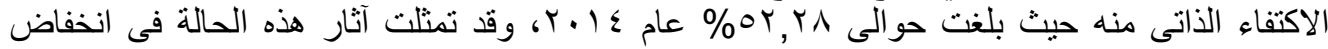

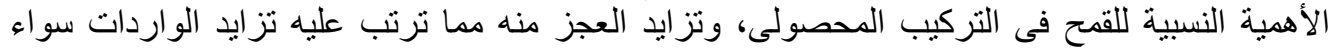

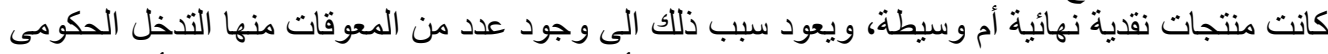

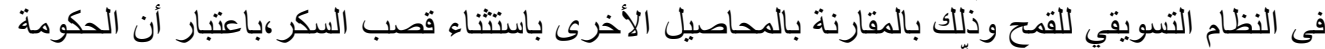

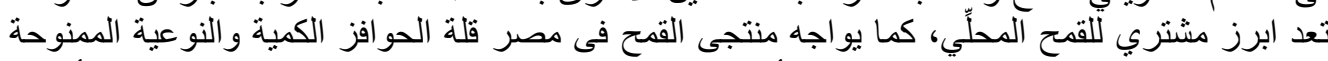

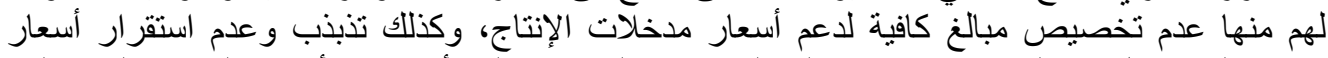

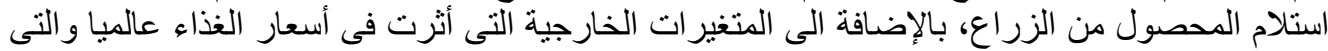

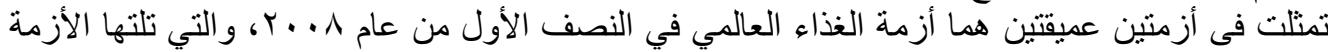

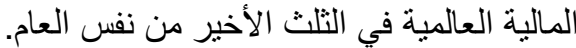

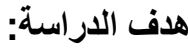

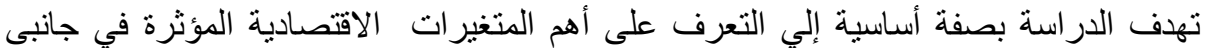

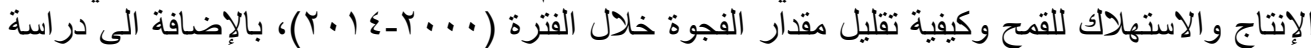

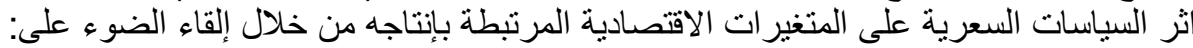

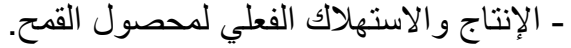

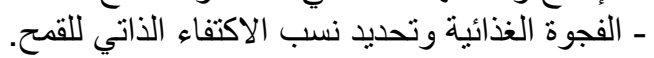
ـ التقدير القياسى للفجوة من محصول الفيد القمح فئ مصر. ـ در اسة اثر أزمة الغذاء العالمي في النصف الأول من عام ^ ... على قيمة الناتج من محصول القمح.

Fayoum J. Agric. Res. \& Dev., Vol. 30, No.1, January, 2016 
$\vee q$

ـ تقدير الأسعار المزرعية لمحصول القمح وفقا لبعض البدائل السعرية ومقارنتها بالأسعار المزرعية

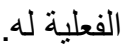

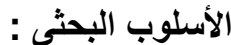

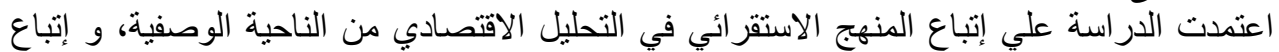

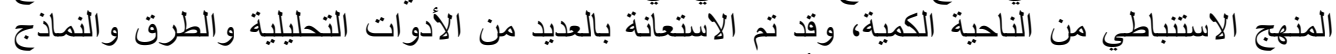

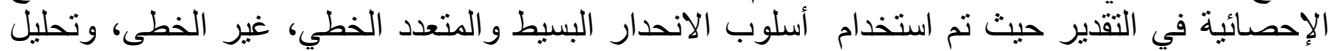

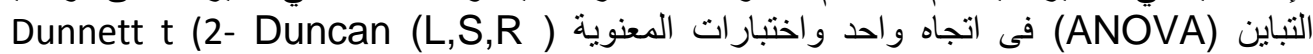
(sided)

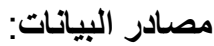

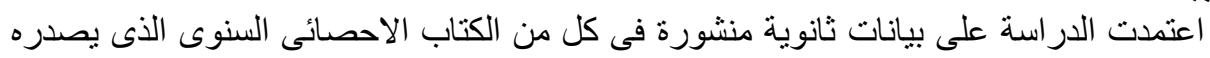

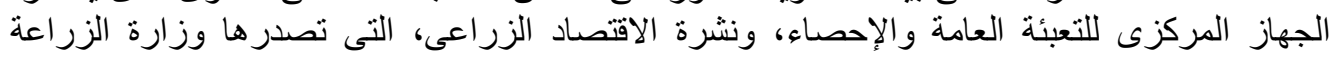

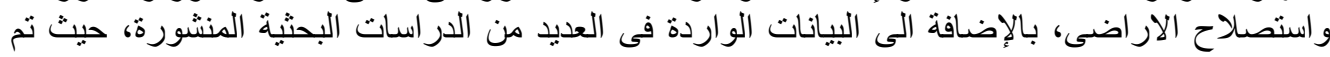

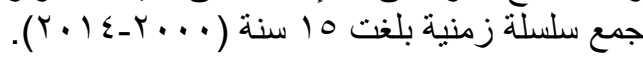

نتائج الاراسة

أولا: واقع الإتتاج والاستهلاك الفعلي و الفجوة الغذائية لمحصول القمح فى مصر:

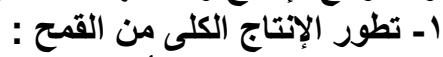

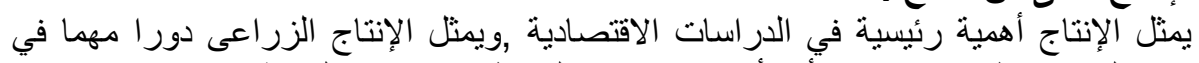

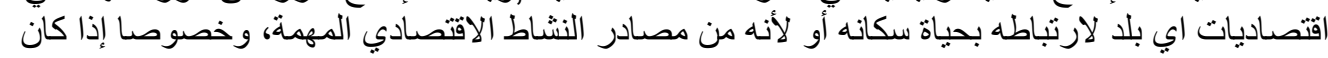

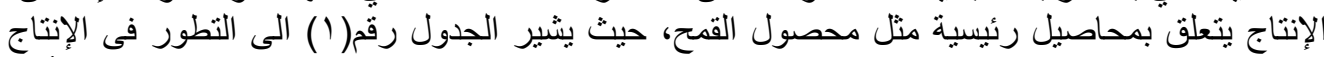

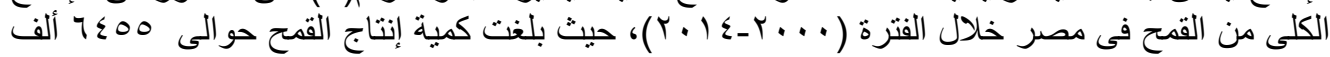

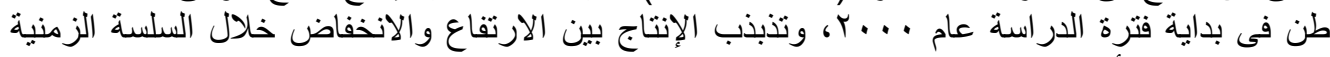

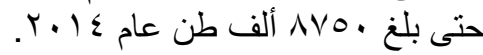
جدول (1) الإتتاج والاستهلاك و والفجوة ونسب الاكتفاء الذاتي والاعتماد علي الخارج لمحصول

\begin{tabular}{|c|c|c|c|c|c|}
\hline \multicolumn{6}{|c|}{ 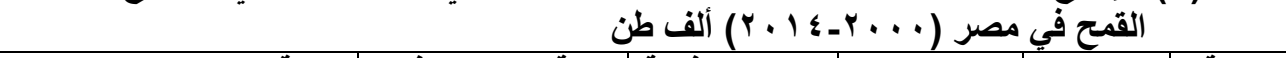 } \\
\hline نسبة الاعتماد علي الخارج & نسبة الاكتفاء الذاتي & الفجوة الغذائية & الاستهلاكك & الإنتاج & السنة \\
\hline$\leq 1, \leqslant 7$ & $0 \wedge, 0 \leq$ & $\leq 0 \vee 1-$ & $11 \cdot 17$ & $7 \leqslant 00$ & $r \ldots$ \\
\hline$\varepsilon r, \cdots$ & ov, , & 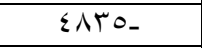 & $11 r \leq \varepsilon$ & $7 \leq .9$ & 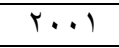 \\
\hline$\leqslant 1,0 Y$ & $0 \wedge, \leqslant \Lambda$ & ¿०VY- & $11 \cdot 11$ & $7 \leq \varepsilon$ & $r \cdots r$ \\
\hline$r v, r q$ & $\pi Y, 71$ & $\varepsilon \cdot 9 \cdot-$ & $1.9 \leq$. & 710. & $r \ldots r$ \\
\hline ro, vo & $7 \varepsilon, Y_{O}$ & r990_ & 11180 & $\vee \backslash \wedge$. & $r \ldots \varepsilon$ \\
\hline$\varepsilon 1,1 \pi$ & $O \wedge, \wedge V$ & $07 \wedge \Lambda$ & IrNYq & $\wedge 1 \leq 1$ & $r \ldots o$ \\
\hline$\sum 1, r \wedge$ & $O \Lambda, V Y$ & $O \wedge / V_{-}$ & $1 \leq .91$ & AYVE & $r \ldots T$ \\
\hline$\varepsilon \leqslant, \leqslant \wedge$ & $00,0 Y$ & $0911-$ & IrYq. & VrVq & $r \ldots V$ \\
\hline$\sum \Lambda, \cdot 7$ & $01,9 \leq$ & VTNI- & 10101 & VqVV & $r \ldots \Lambda$ \\
\hline$\sum \Sigma, \wedge \uparrow$ & $00,1 \leq$ & 794r- & $10 \leqslant 07$ & AOYT & $r \ldots q$ \\
\hline $0 Y, 00$ & $\leqslant V, \leqslant 0$ & V৭r人_ & $101 \cdot V$ & 1179 & $r \cdot 1$. \\
\hline 04,97 & $\leqslant 7, \cdot \varepsilon$ & $9 \wedge 11-$ & IAIAY & NHV & $r+11$ \\
\hline$\leqslant 7,9$. & or, 1 . & $\vee \vee \neg \wedge$ & $1707 \varepsilon$ & ᄉV97 & $r .1 Y$ \\
\hline$\varepsilon r, Y \Lambda$ & $07, V Y$ & $V Y \backslash A_{-}$ & $177 V A$ & $9 \leq 7$. & $r .1 T$ \\
\hline$\varepsilon V, V Y$ & $O Y, Y A$ & V৭AV- & ITVTV & AVo. & $r \cdot I \leq$ \\
\hline$\varepsilon \varepsilon, Y Y$ & $00, \vee \wedge$ & $7 r \cdot 1, \cdot V_{-}$ & $1 \leq \cdot \leq 7, \cdots$ & $V V \leq \varepsilon, q r$ & المتوسط \\
\hline
\end{tabular}

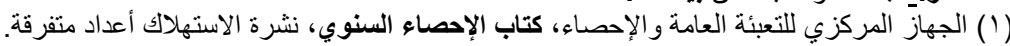

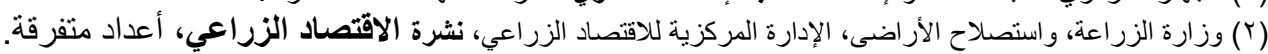

Fayoum J. Agric. Res. \& Dev., Vol. 30, No.1, January, 2016 
$\Lambda$.

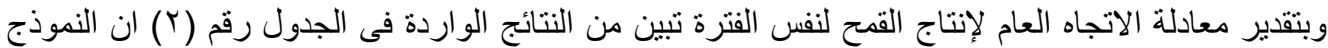

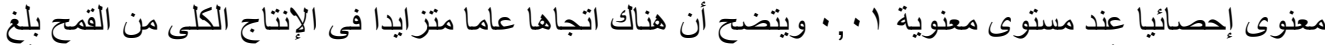

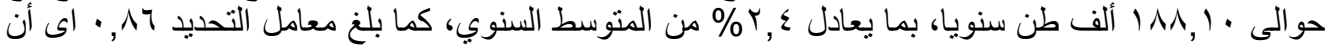

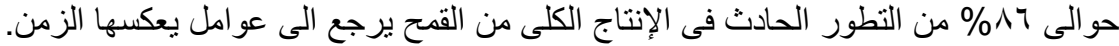

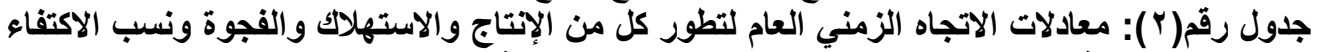

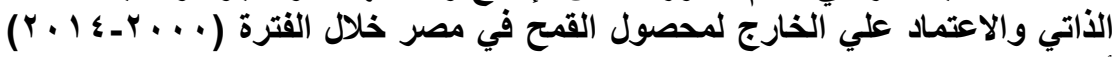

ألف طن الآن

\begin{tabular}{|c|c|c|c|c|c|c|}
\hline \multirow{2}{*}{ iر } & \multirow{2}{*}{ ف } & \multicolumn{2}{|c|}{ التغير السنوي } & \multirow{2}{*}{ متوسط الظرة } & \multirow{2}{*}{ نموذج الاتجاه الزمني العام } & \multirow{2}{*}{ البيان } \\
\hline & & معدل \% & مقدار & & & \\
\hline$\cdot, \wedge \uparrow$ & ${ }^{* *} r \mu$ & $r, \varepsilon$ & $1 \wedge \wedge, 1$. & $V V \leq \varepsilon, 9 \Gamma$ & صنه = & الإنتاج \\
\hline$\cdot, 94$ & ${ }^{* *} \wedge T$ & $r, v$ & Or. & $1 \leqslant \cdot \leqslant 7$ & صـ & الاستهلاك \\
\hline$\cdot, \wedge \uparrow$ & ${ }^{* *} r v^{2}$ & $0, Y 7$ & & 7 & صد^ = & الفجوة \\
\hline$\cdot, \leqslant 9$ & $* * q, r_{0}$ & $1, \Gamma_{-}$ & $\cdot, V T_{-}$ & $00, \vee \wedge$ & صس & نسبة الاكتقاء \\
\hline$\cdot, O Y$ & $* * 1 \cdot, r$ & 1,7 & $\cdot, V T$ & $\varepsilon \varepsilon, Y Y$ & ص ص^ه = & علي الخارج \\
\hline
\end{tabular}

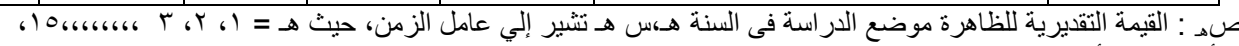

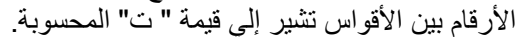

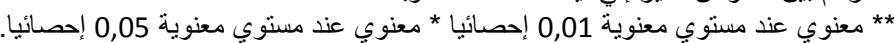

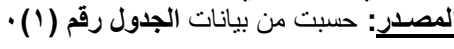

r - تطور الاستهلاك الكلى من القمح:

تشير بيانات الجدول رقم (1) الإني تزل ايد الكمية المستهلكة من القمح على مستوي الجمهورية خلال

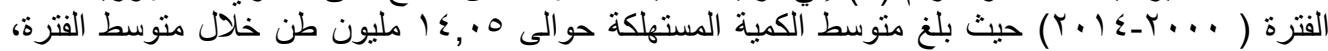

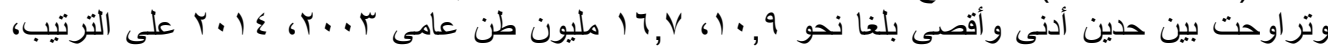

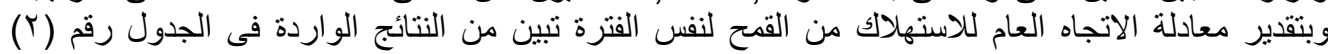

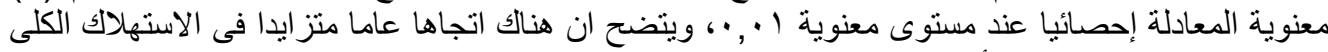

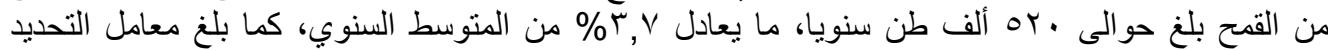

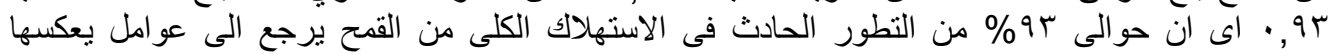

r- تطور الفجوة الغذائية من القمت:

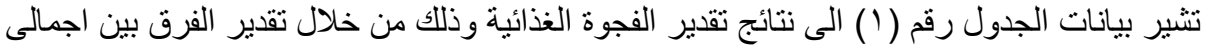

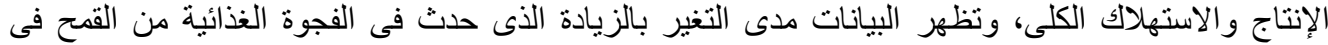

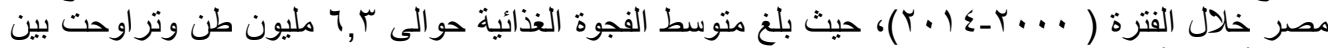

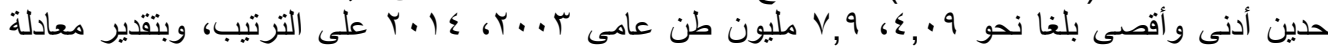

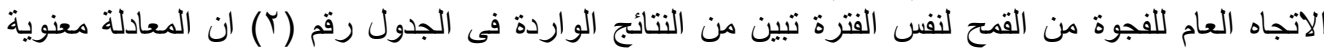

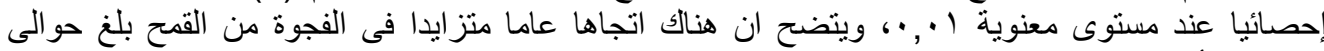

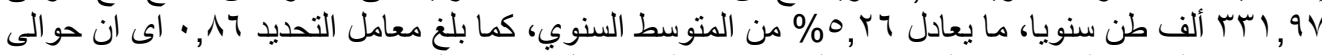

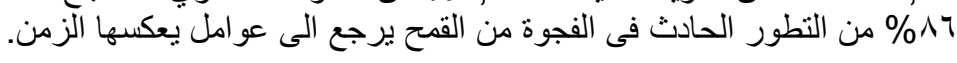

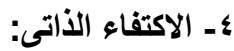

Fayoum J. Agric. Res. \& Dev., Vol. 30, No.1, January, 2016 
A

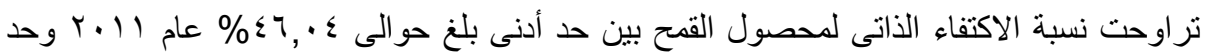

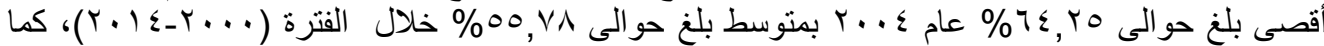

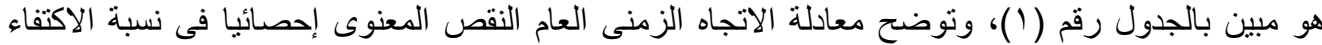

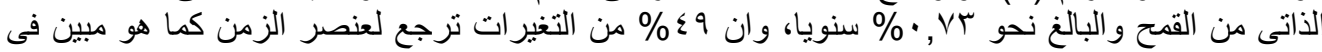

0ـ مؤشر نسبة الاعتماد علي الخارج:

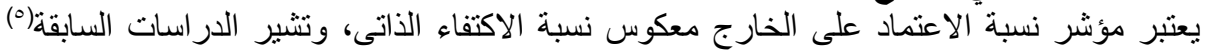

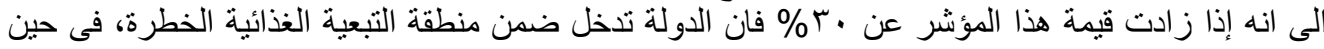

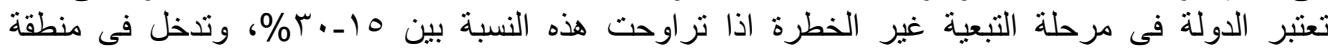

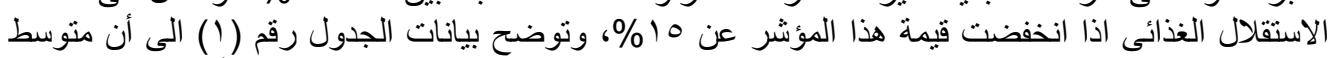

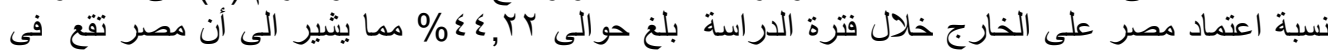

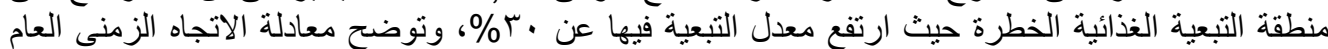

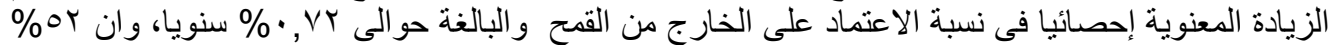

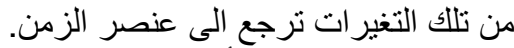

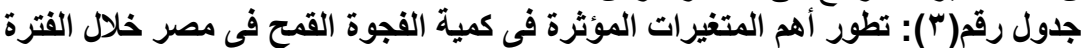
$(r+1 \leq-r \ldots)$

\begin{tabular}{|c|c|c|c|c|c|c|c|c|c|}
\hline |قيمة الناتج من & |ألف فداحة & طالفدانية & جنيه / المزرعى طن & مليمة فجوة & نليون السكان & جنيه للطن العالمى & قليمة الناتج & مليون جنيمة & السنوات \\
\hline$\varepsilon \leqslant 77, \wedge \uparrow$ & rะTr & $T, T V$ & 794 & YIYT & $\pi T_{1}$ & מTO & $r \varepsilon .1 \ldots$ & TrqVY & $r \ldots$ \\
\hline$\leqslant \leqslant \vee q, \wedge q$ & $r T \leqslant Y$ & $T, 7 V$ & 799 & $1 V \cdot V$ & Tr. & $7 \vee 9$ & rosv.. & $r \leqslant V 00$ & $r \ldots l$ \\
\hline$\sum \neg \mu \cdot, \Pi$ & $r \leqslant 0$. & $r, V$ & 519 & $r \cdot 1 \varepsilon$ & $T \leqslant, V$ & $7 V$. & 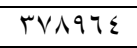 & $r \wedge \Lambda \cdot \varepsilon$ & $r \ldots r$ \\
\hline 0199,10 & ro.7 & $r, V r$ & vo9 & $r .79$ & $77, \cdots$ & $9 . r$ & $\varepsilon 1 \vee 019$ & $\varepsilon .0 \leqslant$. & $r \ldots r$ \\
\hline$V 170,7 \varepsilon$ & ro.o & $Y, V T$ & $99 \wedge$ & $\varepsilon \leqslant Y \wedge$ & $T V, r$ & $1 . r r$ & $\sum \wedge O r \leqslant Y$ & $\sum V T V A$ & $r \ldots \varepsilon$ \\
\hline $91 \cdot 1,7 \leq$ & r910 & $r, V r$ & 1111 & 04.1 & $V \cdot, V$ & $9 \leqslant 1$ & 041011 & 71710 & r... \\
\hline $9 M 17,0 r$ & $r \cdot T \varepsilon$ & $r, V$ & $11 Y 7$ & 0049 & $V Y, Y$ & $90 \leqslant$ & $\pi \vee V \vee \leq \varepsilon$ & 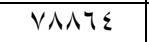 & $r \ldots T$ \\
\hline $1 \wedge \wedge 17, \leqslant 0$ & TVIT & $r, V r$ & roo. & $1 \wedge 19$ & $V r, T$ & $1 \leqslant 90$ & $V \Sigma \varepsilon V \wedge V$ & 91 Y07 & $r \ldots V$ \\
\hline$r \cdot r 70, Y \wedge$ & rqY. & $r, V$ & $171 \%$ & 110.9 & $V O, Y$ & $194 \wedge$ & $1900 . r$ & $\mid \leq \Psi 1 \cdot V$ & $r \ldots \lambda$ \\
\hline $10 \leqslant r 0,10$ & $\Gamma ! \leqslant V$ & $r, V q$ & 1111 & 107ร & $V 7,9$ & YIYV & $1 . \leqslant Y 100$ & $1 T \leq 0 \wedge 9$ & $r \ldots q$ \\
\hline $17 \Lambda \cdot \varepsilon, 1 \leq$ & $r \ldots 1$ & $r, r q$ & $r T \leq \varepsilon$ & IYITV & $\vee \wedge, \vee$ & rYqI & $1 \% .709$. & $10 \leqslant 10$. & $r \cdot 1$. \\
\hline$r 1 \cdot 79, \lambda 1$ & $r \cdot \leqslant q$ & $r, V \varepsilon$ & rolV & 19.19 & $\Lambda, r$ & $r \cdot r q$ & $1 \mathrm{KV} / 1.7$ & IANTO| & $r+11$ \\
\hline YYTTV,Yq & T17. & $r, V A$ & YOVY & T.ONE & $\Lambda \varepsilon, 7$ & TOTK & lov00YV & IVAOIY & $r .1 r$ \\
\hline$r \leq r V \wedge, \leqslant Y$ & TrVV & $r, \wedge 1$ & roVY & 51791 & $\wedge \neg, r$ & rlor & IVOTYOY & 19VVY. & $r .1 T$ \\
\hline$r \leqslant \varepsilon V r, V 0$ & $r \Sigma) \leqslant$ & $r, \wedge \varepsilon$ & TVqV & rT70r & 19,01 & 1794 & 19.110. & $190 Y \wedge$ & $r .1 \leq$ \\
\hline $1 \Gamma \wedge 91, r 7$ & YAVT,YT & $r, v 1$ & $1709, \mathrm{VT}$ & $1.9 \leq \vee, 7$ & $V^{\top}, \wedge 0$ & $10 \mathrm{r}, 94$ & $9 . \wedge 947,7$ & $1.0994,04$ & المتوسط \\
\hline
\end{tabular}

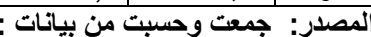

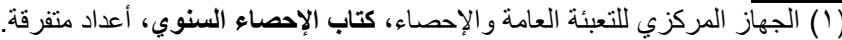

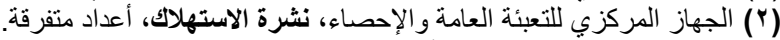

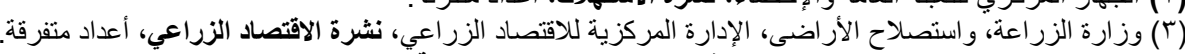

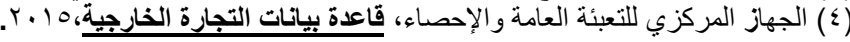

أوضحت النتائج الواردة فى الجدول رقم (ع) ان كمية الفجوة من محصول القمح تأترت معنويا بكل

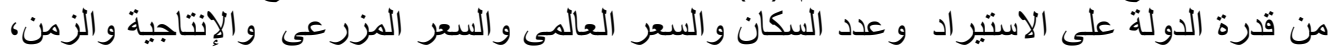

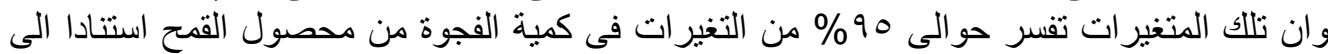

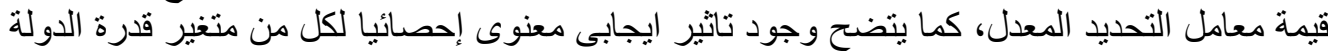

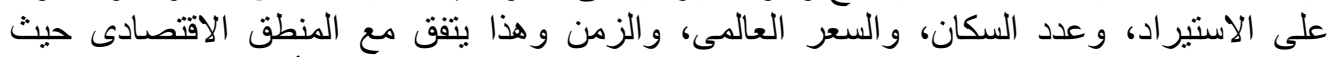

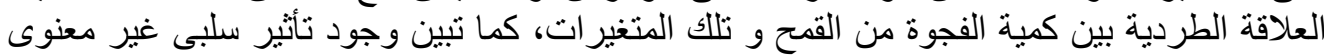
إحصائيا لكل من متغير السعرد المزرعى، والإنتاجية، والعلاقة العكسية هنا تثفق مع المنية المنطق

Fayoum J. Agric. Res. \& Dev., Vol. 30, No.1, January, 2016 
$\Lambda r$

الاقتصادى فبزيادة السعر المزرعى كحافز للإنتاج تنخفض الفجوة، وكذلك بزيادة الإنتاجية الفدانية

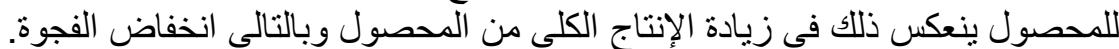

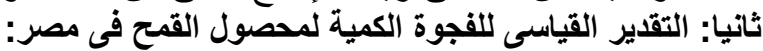

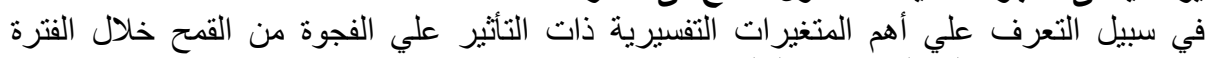

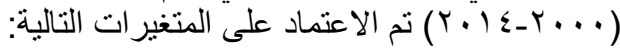
ـ مقدرة الدولة علي استير اد القمح وقد أمكن التعبير عنها من خلال الأهمية النسبية لقيمة الصادرات الي الناتج المحلي. - عدد السكان و هو يعكس إضافة طلب جديد علي المحصول مع مرور الزمن.

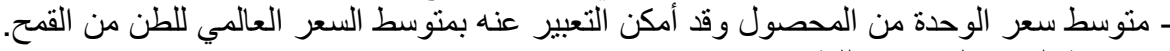
ـ - منوسط السعر المزرعى للطن.

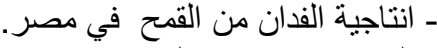

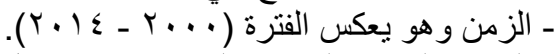

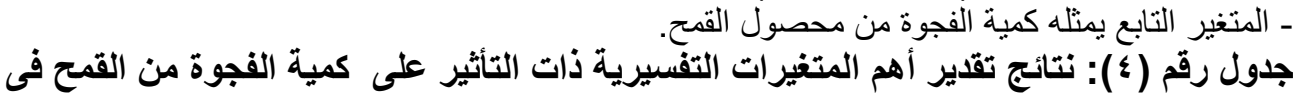

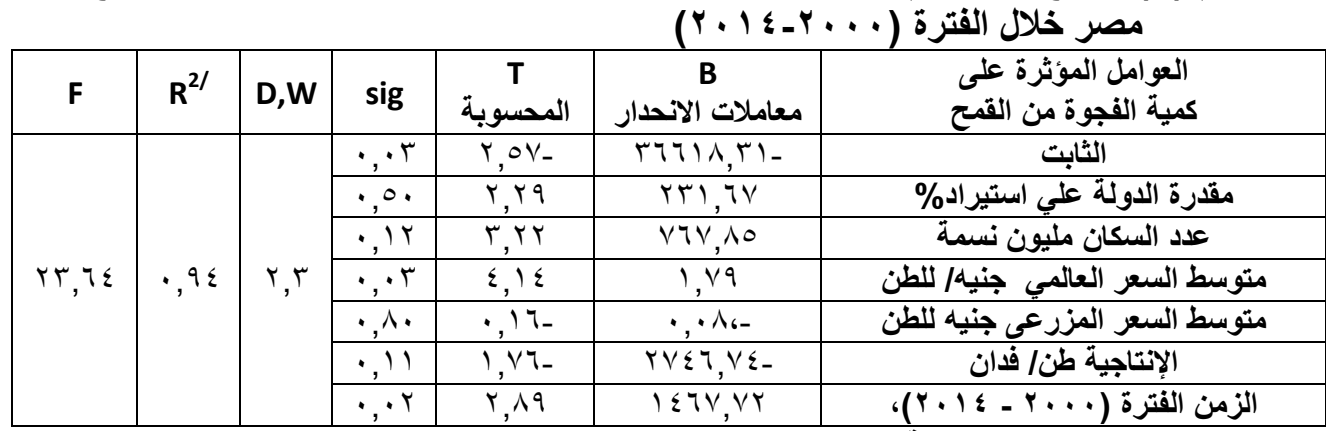

المصدر: حسبت من بيانات الجدول رقم (r).

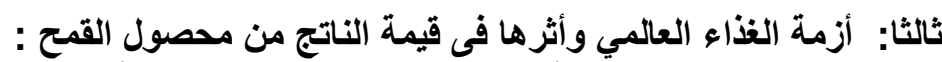

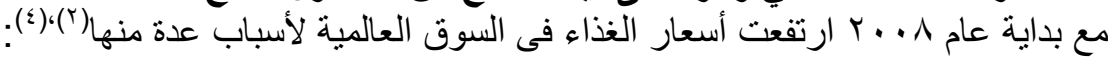

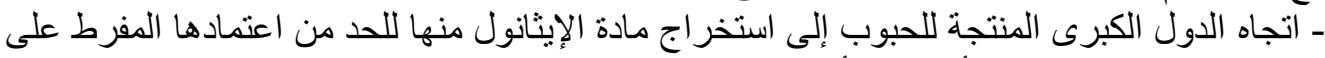

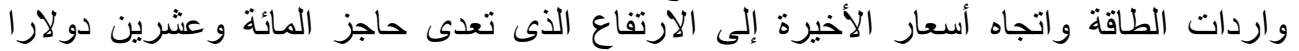

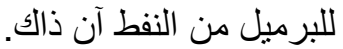

- نقص إنتاج الحبوب لأربعة مو اسم متتالية لعو امل بيئية ومناخية منها:

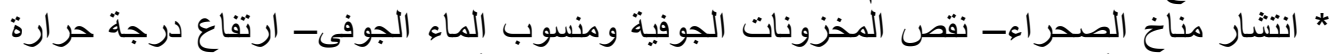

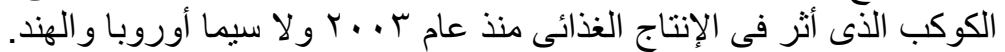

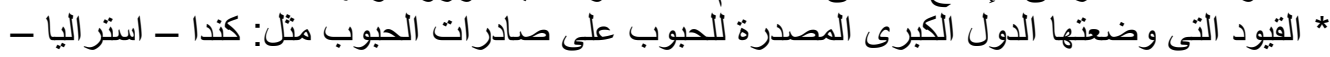

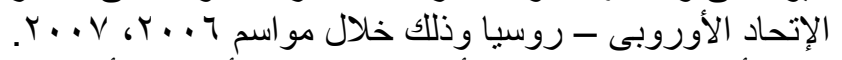

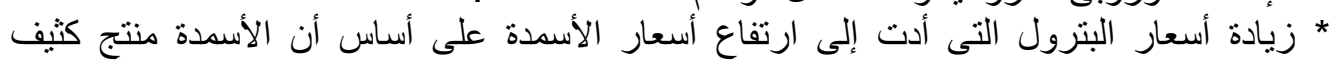

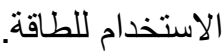

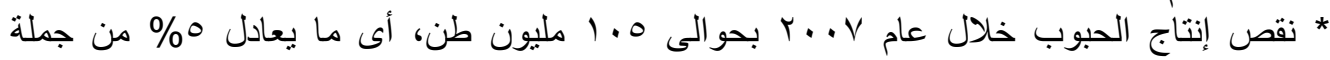

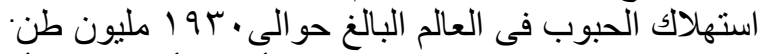

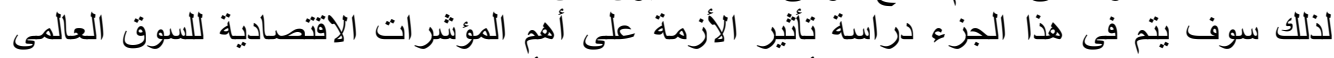

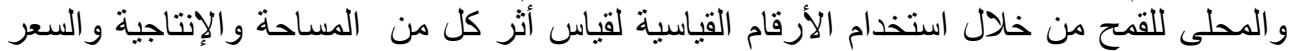

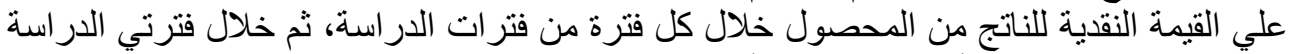

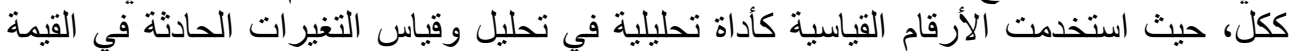

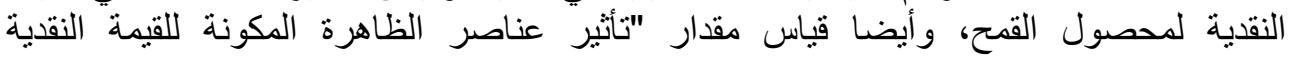

Fayoum J. Agric. Res. \& Dev., Vol. 30, No.1, January, 2016 
$\Lambda r$

للمحصول (D) والتي تتمثل في المساحة المزروعة (A) ومتوسط إنتاج الفدان (B) والسعر المزرعي (C) علي التغيرات الحادثة في القيمة النقدية للمحصول، وذلكة ولك باستخدام المعادلات

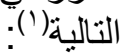

$=A_{i} B_{i} C_{i} / A_{o} B_{0} C_{o} * 100$

الرقم القياسي لأثر عناصر الظاهرة

$=A_{i} B_{i} C_{i}-A_{0} B_{0} C_{0}$

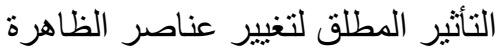

$=A_{i} B_{0} C_{0}-A_{o} B_{o} C_{0}$

$=A_{i} B_{0} C_{0} / A_{0} B_{0} C_{0}^{*} 100$

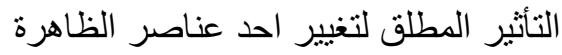
الرقم القياسي لأثر تغيير احد عناصر النير الظاهرة

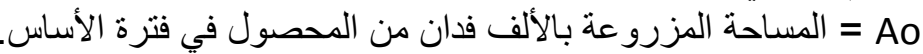

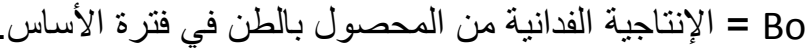
Co

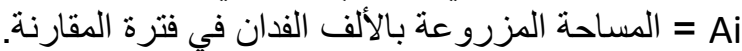
Bi Ci l السعر المزرعي الجاري للطن في فترة المقارنة.

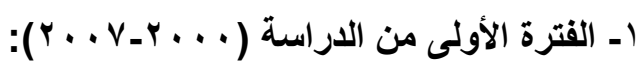

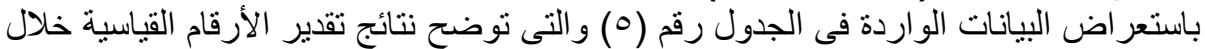

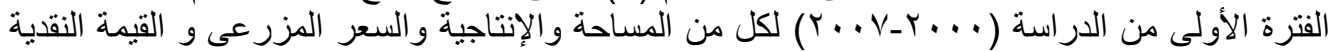

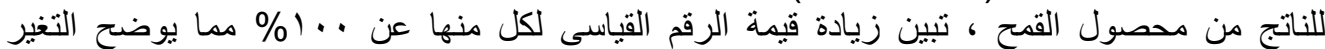

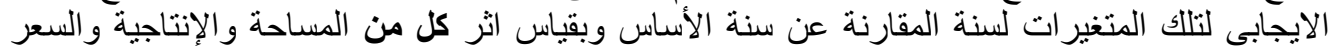

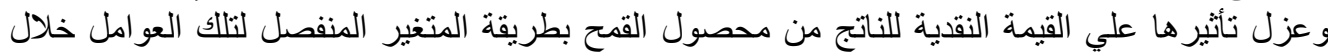

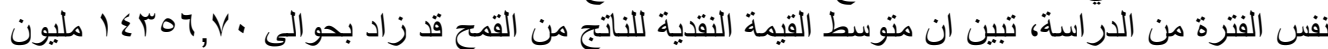

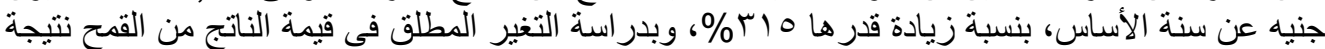

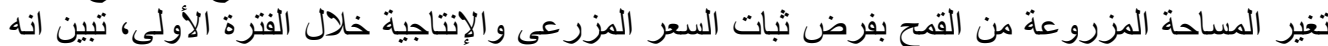

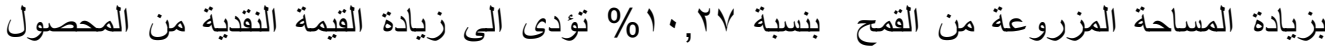

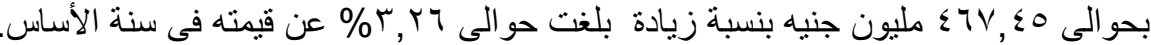

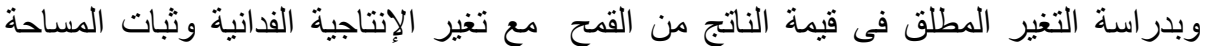

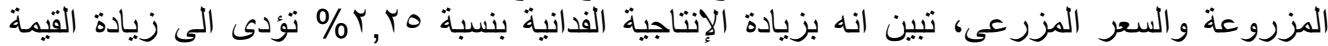

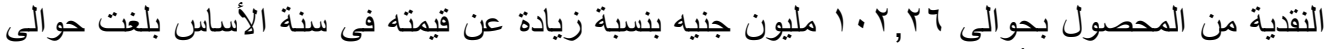

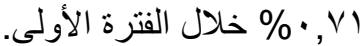

جلول رقم(•): نتائج تحليل اثر تغير المساحة والإنتاجية والسعر المز رعى على القيمة النقية

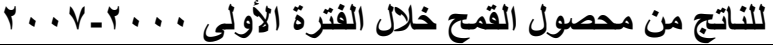

\begin{tabular}{|c|c|c|c|c|c|c|}
\hline \multicolumn{7}{|c|}{ 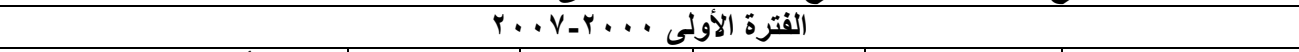 } \\
\hline \multicolumn{2}{|c|}{ تأثير تغير الظاهرة } & \multirow[b]{2}{*}{ الرقم القياسى } & \multirow{2}{*}{ سنة المقارنة } & \multirow{2}{*}{ سنة الأساس } & \multirow[b]{2}{*}{ وحدة القياس } & \multirow[b]{2}{*}{ عناصر الظاهرة } \\
\hline النسبى \% & مليون جنيه & & & & & \\
\hline$r, r q$ & $\leq T V, \leqslant 0$ & $11 \cdot, r V$ & rVIT & $r \leqslant \pi r, \cdot \cdot$ & ألف فدان & A المساحة A \\
\hline$\cdot, \mathrm{V})$ & $1 \cdot Y, Y T$ & I. Y, YO & $\mathrm{r}, \mathrm{VT}$ & $r, T V$ & ط & الإتتاجية Y \\
\hline 10,11 & IYYIA,T. & $r 41,0$. & roo. & $795, \cdots$ & جنيه/طن & السعر المزرعى P \\
\hline$r, r^{\top}$ & I $\leqslant r 07, \vee v$. & $\varepsilon 10, \varepsilon \wedge$ & $1 \wedge 9 \cdot v, \varepsilon r$ & $\varepsilon 00 \cdot, \mathrm{V} \varepsilon$ & مليون جنيه & قيمة الناتج PV \\
\hline
\end{tabular}

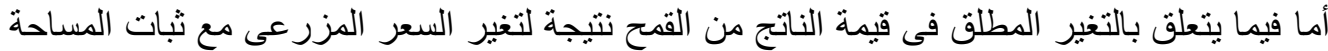

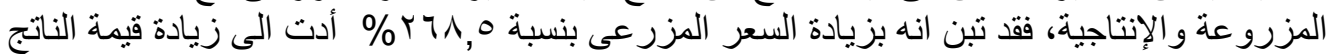

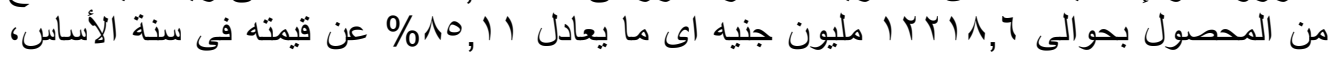

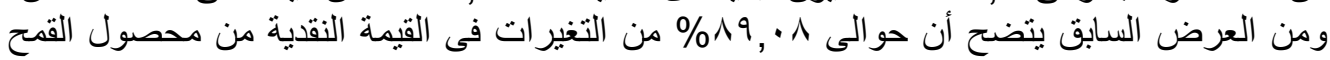

Fayoum J. Agric. Res. \& Dev., Vol. 30, No.1, January, 2016 
$\wedge \varepsilon$

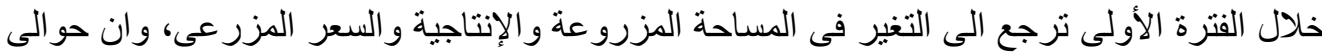

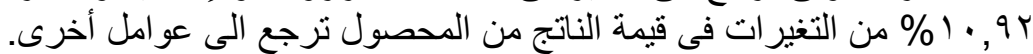

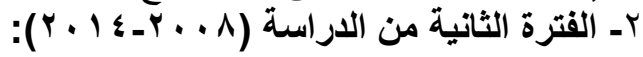

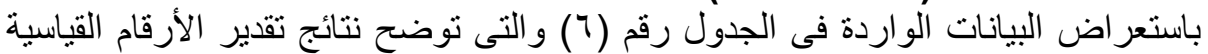

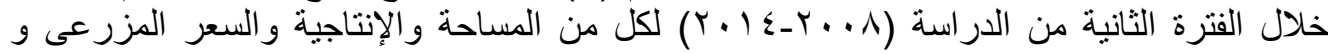

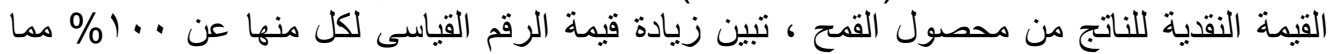

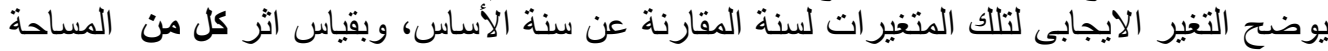

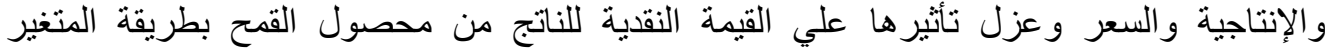

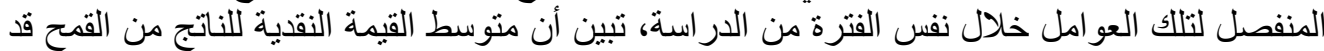

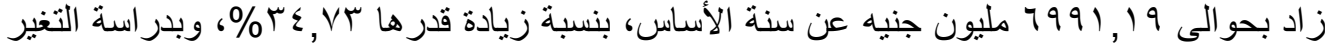

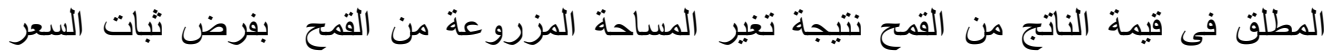

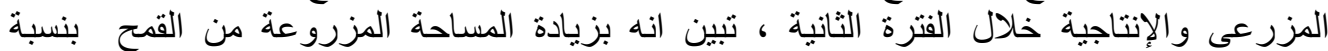

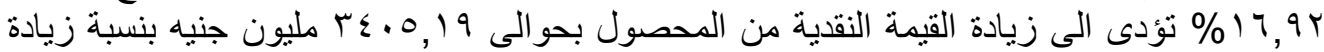

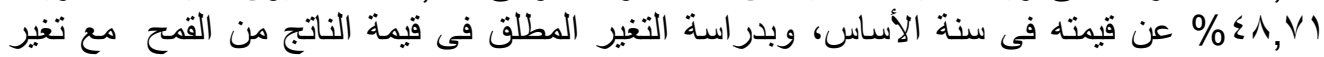

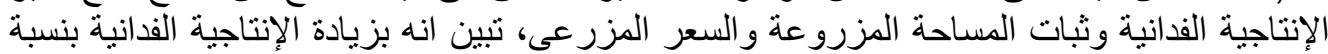

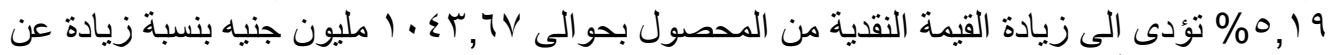

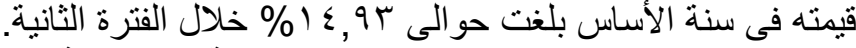
جلول رقم(T): نتائج تحليل اثر تغير المساحة والإتتاجية والسعر المزرعى على القيمة النقاية

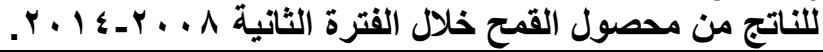

\begin{tabular}{|c|c|c|c|c|c|c|}
\hline \multicolumn{7}{|c|}{ 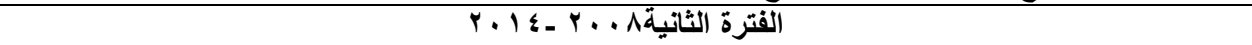 } \\
\hline \multicolumn{2}{|c|}{ تأثير تغير الظاهرة } & \multirow[b]{2}{*}{ الرقم القياسى } & \multirow{2}{*}{ سنة المقارنة } & \multirow{2}{*}{ سنة الأساس } & \multirow[b]{2}{*}{ وحدة القياس } & \multirow[b]{2}{*}{ عناصر الظاهرة } \\
\hline 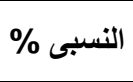 & مليون جنيه & & & & & \\
\hline$\{\Lambda, \mathrm{V}$ & $r \varepsilon .0,19$ & 117,94 & $r \xi 1 \varepsilon$ & rqY. & ألف فدان & | المساحة A \\
\hline $1 \varepsilon, 94$ & $1 \cdot \varepsilon r, T V$ & $1.0,19$ & $r, \wedge \varepsilon$ & $r, r$ & ط & الإنتاجية Y \\
\hline YV,OY & $19 Y 4, Y$. & $1.9,07$ & YVQY & TOOT & جنيه/طن & السعر المزرعى P \\
\hline & 7991,19 & $1 \Gamma \varepsilon, V r$ & $T V \| 19, \cdot \varepsilon$ & T.IYV,AO & مليون جنيه & قيمة الناتج PV \\
\hline
\end{tabular}

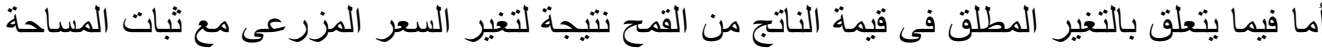

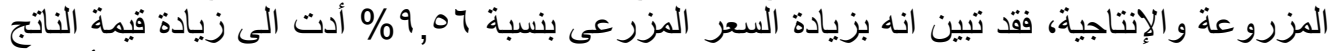

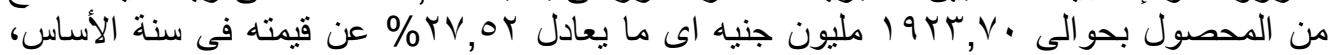

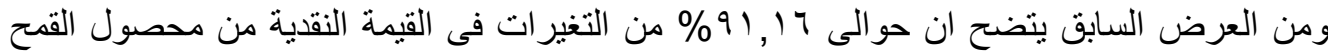

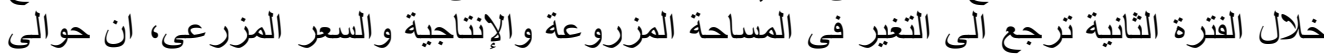

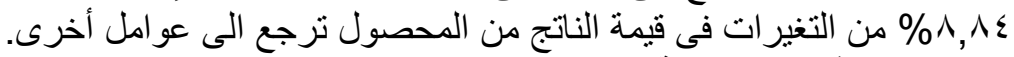

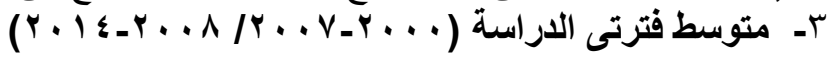

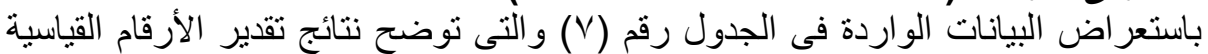

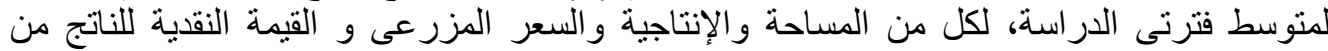

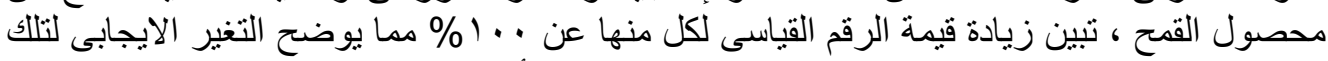

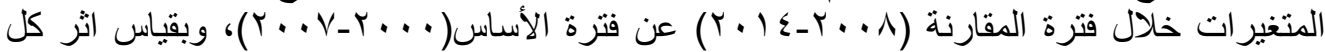

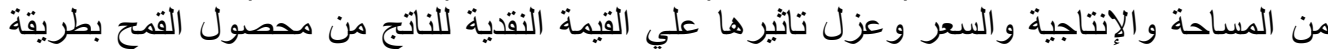

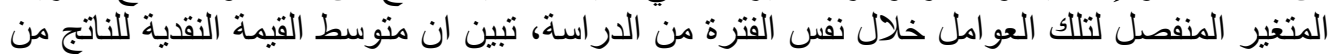

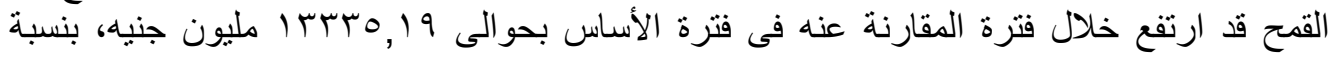

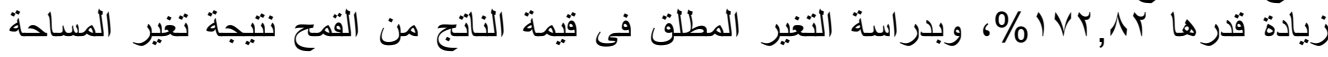

Fayoum J. Agric. Res. \& Dev., Vol. 30, No.1, January, 2016 
المزروعة من القمح بفرض ثبات السعر المزرعى والإنتاجية خلال الفترة الثانية، تبين انه بزيادة الفياة

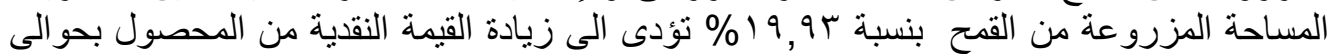
في 1OrV, 9

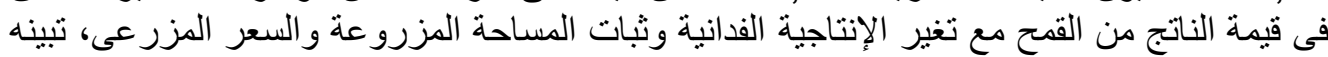

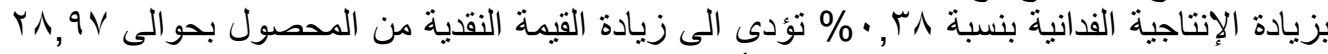

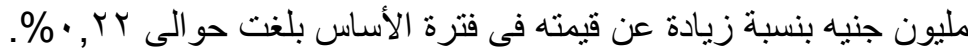

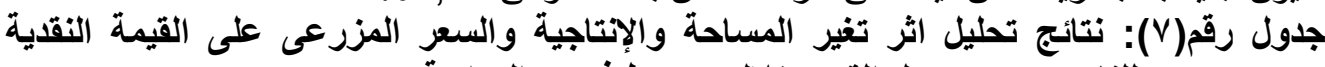

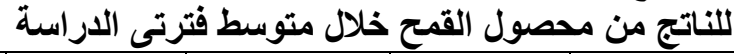

\begin{tabular}{|c|c|c|c|c|c|c|}
\hline \multicolumn{2}{|c|}{ تأثير تغير الظاهرة } & \multirow[b]{2}{*}{ الرقم القياسى } & \multirow{2}{*}{ 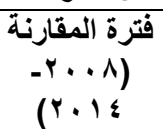 } & \multirow{2}{*}{ 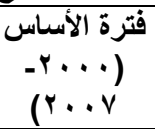 } & \multirow[b]{2}{*}{ وحدة القياس } & \multirow{2}{*}{ الظاهرة } \\
\hline النسبى \% & مليون جنيه & & & & & \\
\hline 11,04 & $104 \mathrm{r}, 19$ & 119,95 & rior,or & YTYA,,$\wedge \Lambda$ & ألف فدان & المساحة A \\
\hline$\cdot, r r$ & $r \wedge, q V$ & $1 \cdots, r \Lambda$ & $r, Y r$ & $r, v)$ & طن & الإتتاجية Y \\
\hline$V T, Y \Lambda$ & $q \vee V Y, \leqslant 9$ & YYT,T & $r \leqslant 0 r, Y)$ & זיד, 1. 1 & جنيه/طن & المزرعى السعر \\
\hline & 1 TrTo, 19 & rVY,AY & $r 1.01,7 \varepsilon$ & $Y V 17, \leqslant 0$ & مليون جنيه & $\begin{array}{l}\text { قيمة الناتج } \\
\text { PV }\end{array}$ \\
\hline
\end{tabular}

المصدر : حسبت من بيانات الجدول رقم(؟).

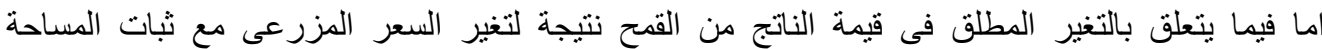

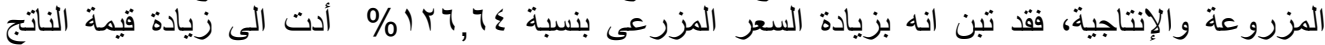

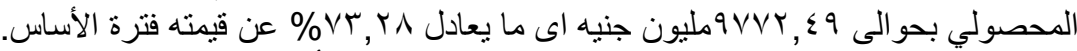

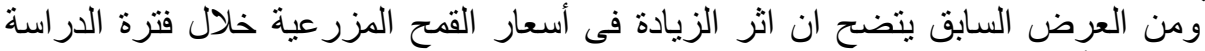

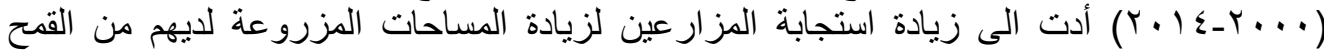

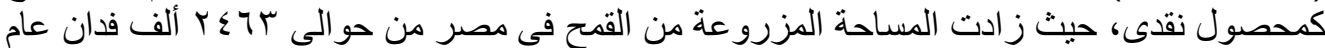

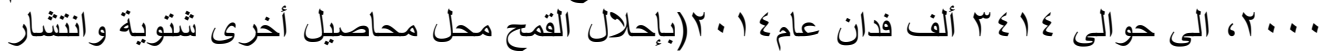

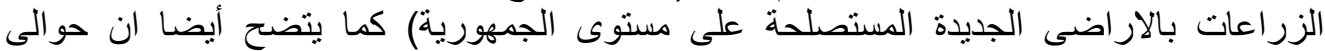

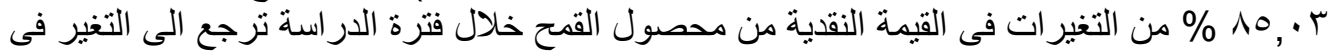

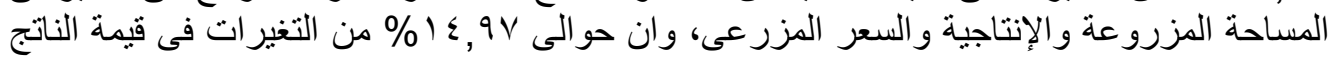
من المحصول ترجع الى عو امل المعل أخرى.

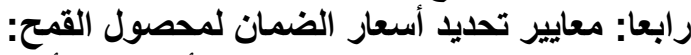

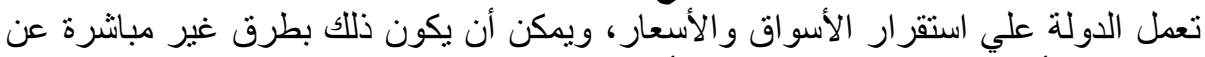

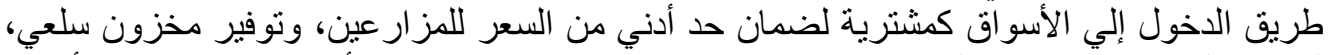

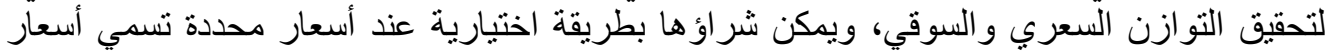

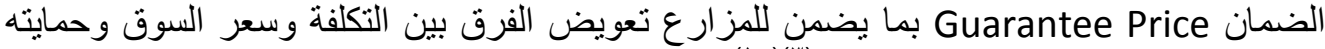
من أي كوارث طبيعية أو أزمات سوقية (־)(· (1).

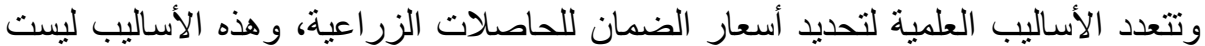

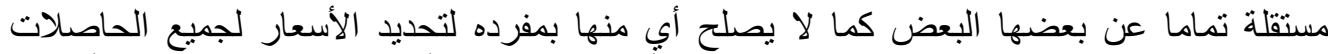

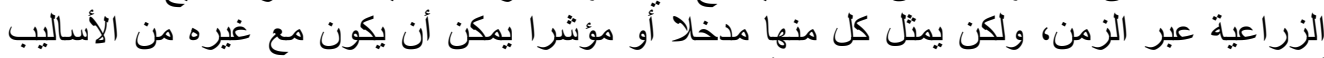
أساسا لتحديد السعر المستهدف ولكن ومن هذه الأساليب: اـ تحديد أسعار الضمان علي أساس تكاليف الإنتاج:

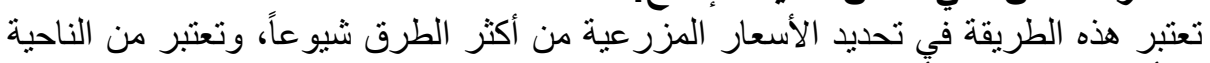
المنطقية من أفضل المعايير و أهمها.

Fayoum J. Agric. Res. \& Dev., Vol. 30, No.1, January, 2016 
$\wedge 4$

وسوف نتناول تقدير الأسعار المزرعية طبقا لمعيار التكاليف باستخدام أكثر من طريقة حسب القيمة

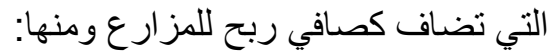

أـ طريقة تكاليف الإنتاج التقليديةً (بإضافة قيمة الإيجار الإنار كصافي ربح للمزارع):

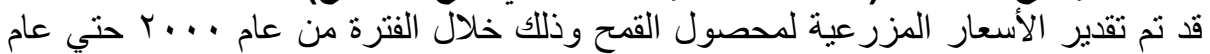

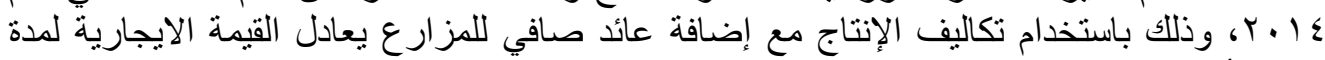

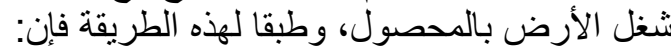
التكاليف الكلية للفذان بما فيها الإيجار + الإيجار ـ قيمة الإنتاج الثانوي للفدان

$$
\text { متوسط إنتاج الفدان من المحصول الرئيسي }
$$

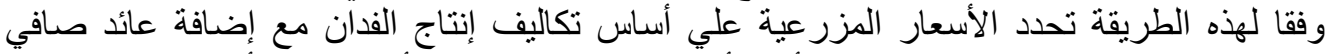

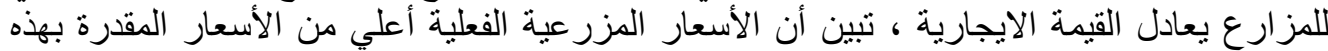

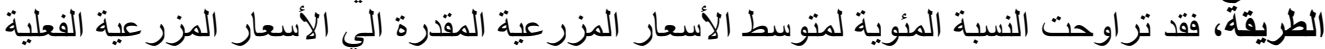

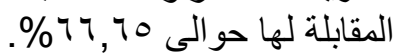
أ- طريقة تكاليف الإنتاج المعدلة (بإضافة ه\%\% مرة و • ه\% مرة من إجمالي التكاليف كصافي ربح

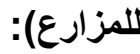
وفقا لهذه الطريقة تحدد الأسعار المزرعية علي أساس تكاليف إنتاج الفذان بما فيها الإيجار

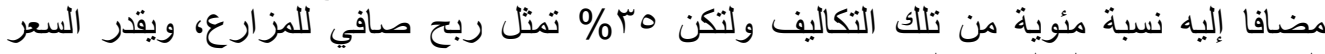
تكاليف الإنتاج للفدان بما فيها الإيجار + هץ\% من التكاليف المزرعي في هذه الحالة كما يلي: من الئية

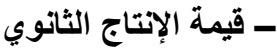

$$
\text { السعر المزرعي للمصول الأساسي = متوسط إنتاج الفدان من المحصول الأساسي }
$$

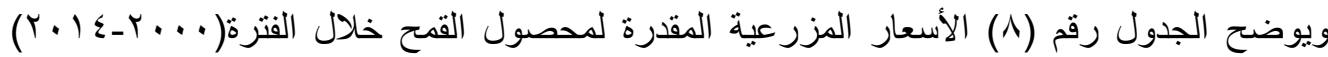

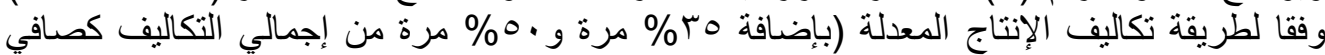

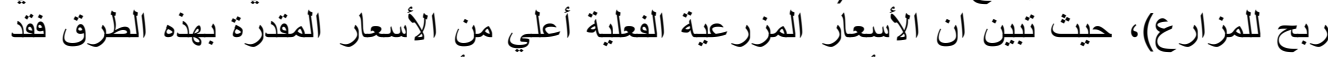

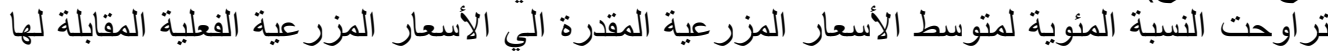

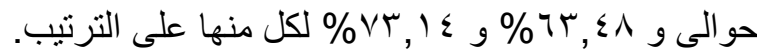

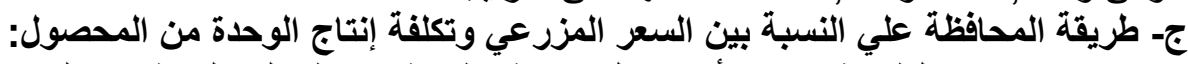

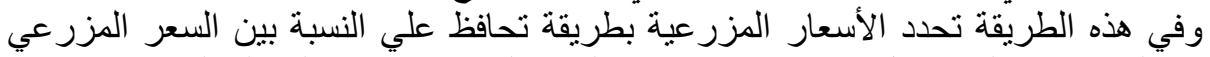
وتكلفة إنتاج الوحدة من المحصول، (بعد استبعاد قيمة الناتج الثانوي من إجمالي التكاليف) كما كانت التئي السي سنة الأسزاس. في سناج الوحة فئة ما = الرقم القياسي لتكلفة إنتاج الوحدة من المحصول في تلك السنة

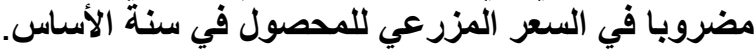

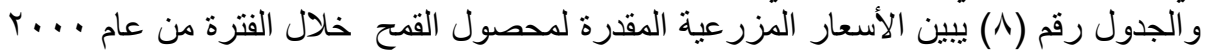

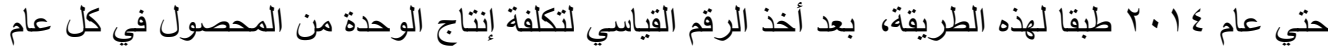

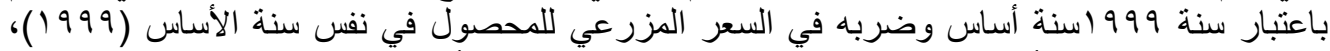

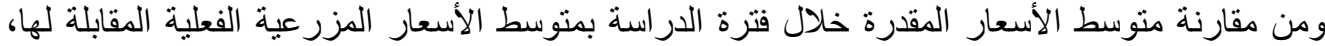

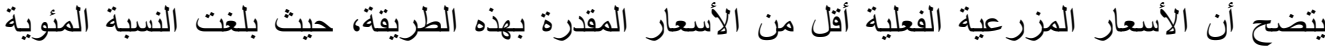

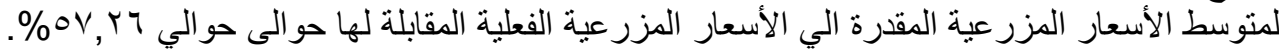

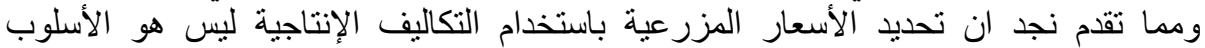

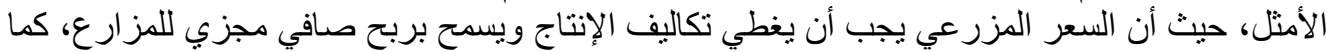

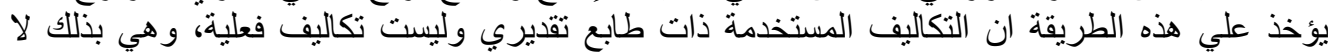

Fayoum J. Agric. Res. \& Dev., Vol. 30, No.1, January, 2016 
$\Lambda \mathrm{V}$

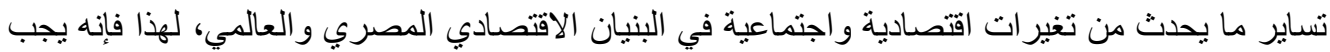

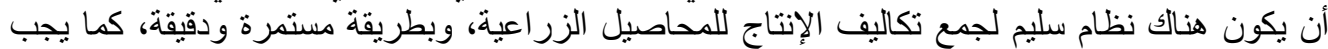

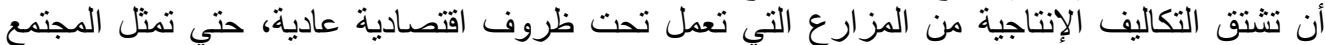

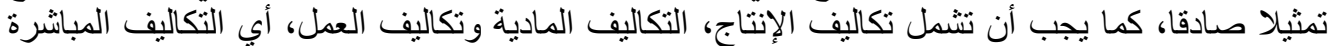

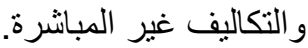

r- تحديد أسعار الضمان طبقا لمعيار أسعار المساواة:

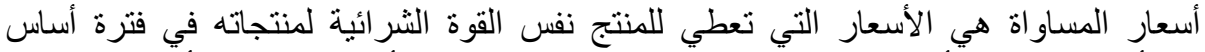

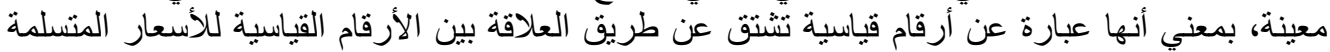

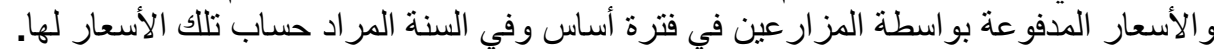

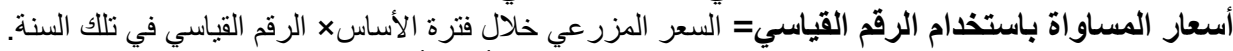

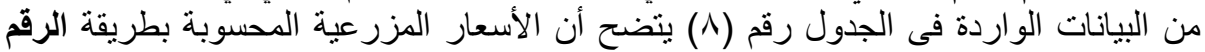

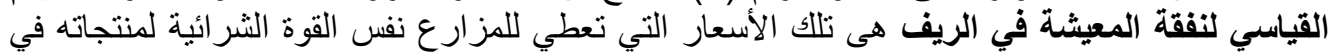

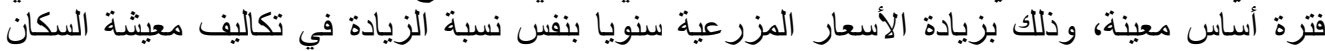

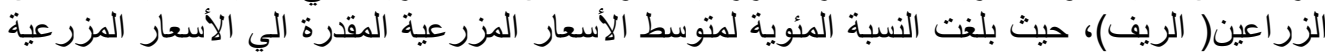

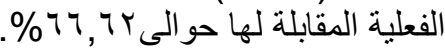

كما يوضح الجدول أيضا أن الأسعار المزرعية المحسوبة بطريقة الرقم القياسي لأسعار الجملة

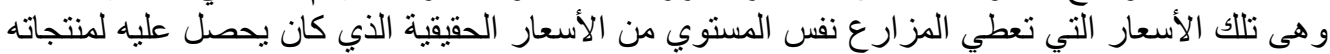

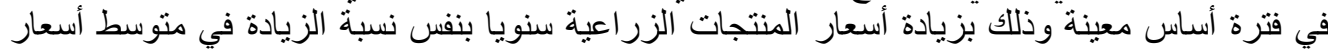

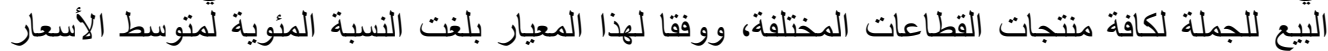

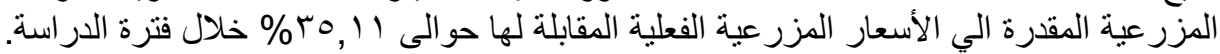

جدول رقم (^): متوسط الأسعار المزرعية المقدرة لمحصول القمح فى مصر و نسبتها من الأسعار

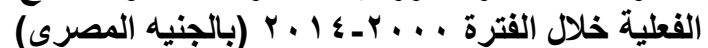

\begin{tabular}{|c|c|c|c|c|c|c|c|c|}
\hline \multicolumn{7}{|c|}{ السعر المزرعى المقدر } & \multirow[b]{2}{*}{ المزلفى المصى } & \multirow[b]{2}{*}{ السنوات } \\
\hline 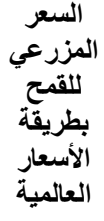 & للاستخدامي الرقين في & الأسبعاستي & المحبة بينة المبلي & اللتكاليفر وفقا & للتكاليف & للتكاليف & & \\
\hline Tro & $r \cdot \lambda, \pi r$ & $7 \times 0, r 9$ & $01 \cdot, \cdot 0$ & $T V \cdot, 0 T$ & $0 \wedge 0, v_{1}$ & $7 r 0,99$ & 794 & r... \\
\hline $7 \times 9$ & $r \mid \xi, Y$ & . A. & $0.9, \cdot r$ & TVT, OT & $0 \wedge v, 9 \Lambda$ & $\pi$ & 799 & $r \ldots 1$ \\
\hline$T$ T. & $r r, \cdot 9$ & $V ז 4,9 \lambda$ & $\sum 9 \wedge, \wedge \uparrow$ & $779, Y r$ & ONr, I & $\pi \cdot, 11$ & 119 & $r \ldots r$ \\
\hline 9.4 & $r \leqslant r, r r$ & $1 \cdot r r, \cdot 1$ & $0 \leqslant r, \cdot 1$ & $V Y V, T 4$ & 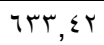 & $T V, \leqslant T$ & yo9 & $r \ldots r$ \\
\hline $1 . r 5$ & $r 9 \wedge, 09$ & IIrT, Ir & $071, \leqslant Y$ & $V Y, Y \varepsilon$ & $T \pi, Y T$ & vis,r. & ११८ & $r \ldots \varepsilon$ \\
\hline
\end{tabular}

Fayoum J. Agric. Res. \& Dev., Vol. 30, No.1, January, 2016 
$\wedge \wedge$

\begin{tabular}{|c|c|c|c|c|c|c|c|c|}
\hline 9 १६। & $\varepsilon 11, v \varepsilon$ & $\| \leqslant \wedge, V Y$ & OTV,IT & var, 97 & 710,11 & $V \Psi \varepsilon, \varepsilon r$ & 1111 & r..o \\
\hline $90 \xi$ & $\{\leqslant r, 19$ & $117 \lambda, 1$. & $7.9,19$ & V & $V \leqslant r, T I$ & $\checkmark \Lambda \nu, Y \Lambda$ & דr & $r . .4$ \\
\hline $1 \leqslant 90$ & $\varepsilon q 1, \Gamma r$ & Irq, 0. & $797, \xi 1$ & $9 \vee 0,9$ & $\Lambda \varepsilon \cdot, \Lambda 1$ & $\Lambda \Lambda \varepsilon, T r$ & roo. & $r \ldots v$ \\
\hline $194 \mathrm{~A}$ & 091,74 & $1100, \mathrm{VA}$ & $q \pi r, r)$ & $T r \cdot 1, T V$ & $11 K 4,9 \varepsilon$ & 1110,94 & $171 Y$ & $r \ldots \lambda$ \\
\hline TIYV & $701, .9$ & $1 \cdot \wedge \cdot, q$. & $1 \cdot 11, r v$ & $1 \leqslant r \mid, r r$ & ITMO,TM & IKrT,r. & $|A| \mid$ & $r \ldots q$ \\
\hline rrqI & $V \Psi, \leq \varepsilon$ & $1 . r \mu, \Pi \Lambda$ & $1 \pi r \xi, \wedge 1$ & $T V \wedge \varepsilon, \bullet \wedge$ & 1004,14 & $177 r, Y \varepsilon$ & $T r \leqslant \varepsilon$ & $r \cdot 1$. \\
\hline$r . r q$ & $\Lambda 11, \cdot Y$ & $1 K \backslash \wedge, T^{\prime}$ & KOV, VV & $V \cdot \varepsilon, Y$. & $\mid \leqslant \Lambda 1, \leqslant \leqslant$ & 1000,11 & Tolv & $r .11$ \\
\hline rorr & AVT, r. & $150, \mathrm{VA}$ & $1 Y \Lambda \cdot, \cdot 9$ & IVVT,OT & $10 r \varepsilon, \lambda$. & $109 r, 11$ & roYY & T.IT \\
\hline rlor & QVY,YY & $1497, \leqslant 7$ & $1 \leqslant \pi 1,9 \wedge$ & $19 \leqslant \leqslant, \leqslant \wedge$ & $17 \Delta V, A r$ & $|V| r, \Lambda \mid$ & roYr & $r .1 Y$ \\
\hline 1794 & $1 . V, Y Y$ & $1 \leqslant 07,74$ & $r \leqslant \leqslant r, \wedge r$ & YIYV, YY & $\mid \wedge O \wedge, \leqslant 0$ & $1 \wedge 70, \leqslant 9$ & TYqY & r.lई \\
\hline $104 \lambda, 94$ & $0 \wedge r, T T$ & $11.0,10$ & $90 \cdot, \leqslant r$ & $|K|\{, \cdot$ & $1.0 \%, \pi$ & $11 \cdot \varepsilon, 71$ & $1709, \mathrm{VT}$ & المتوسط \\
\hline 97,01 & $r 0,11$ & זד, דו & or, ru & $v \pi, 1 \varepsilon$ & $\pi, \leqslant \wedge$ & 77,07 & $1 .$. & من الفقلى \\
\hline
\end{tabular}

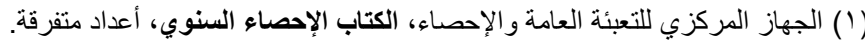

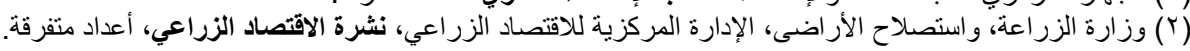

r- تحديد أسعار الضمان طبقا لمعيار الأسعار العالمية المعادلة (فوب- سيف):

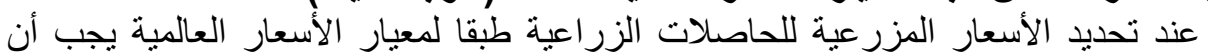

يؤخذ في الاعتبار عاملين أساسيين هما:

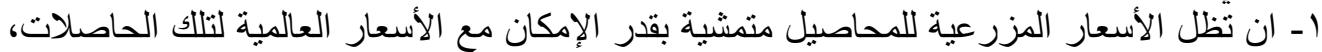

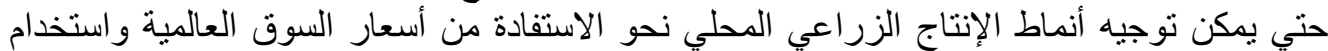

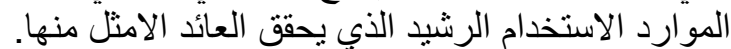

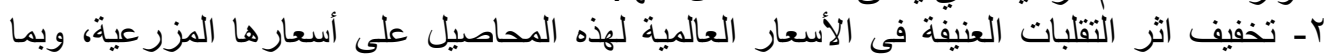

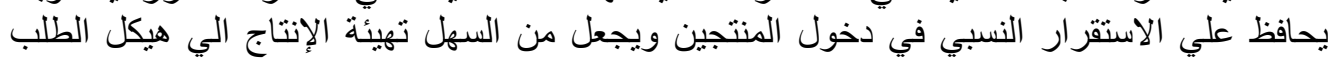

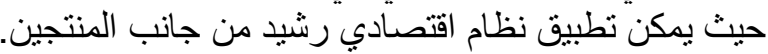

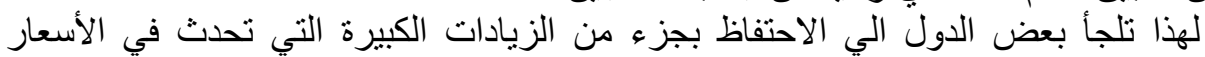

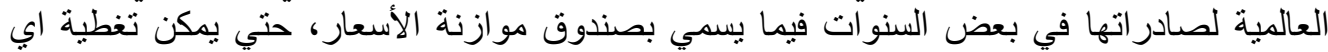

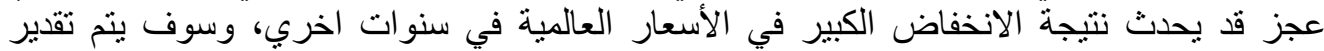

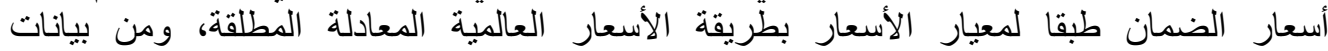

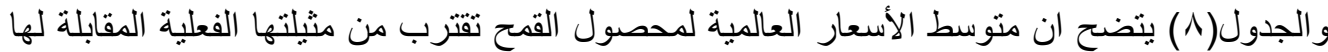

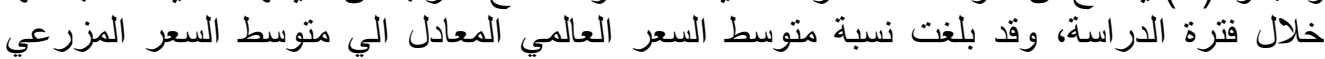

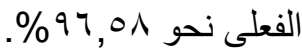

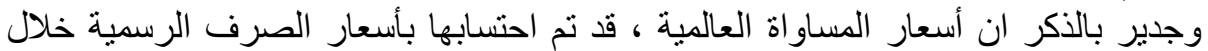

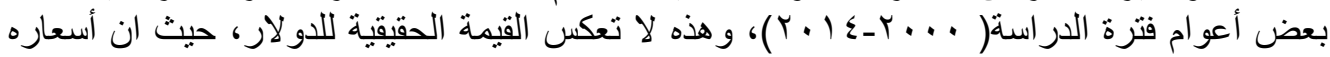

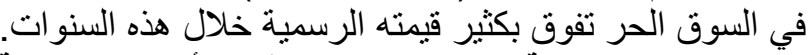

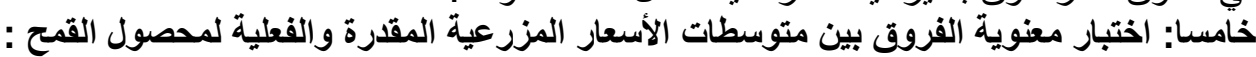

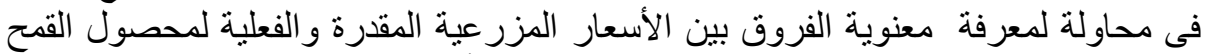

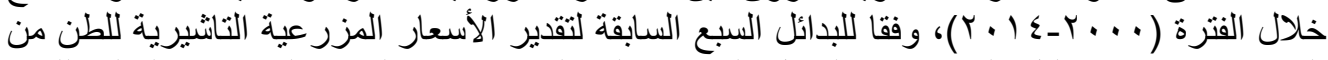

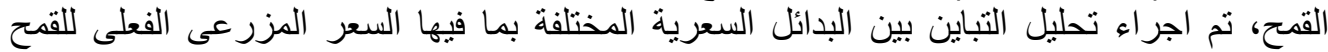

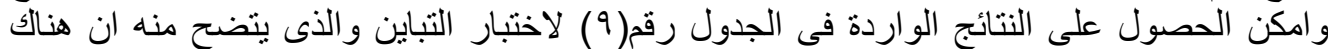

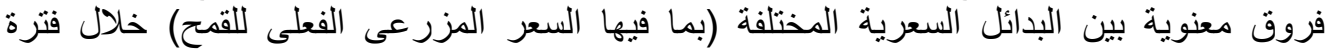

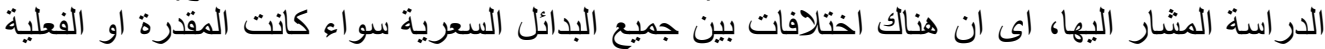

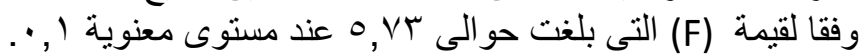

Fayoum J. Agric. Res. \& Dev., Vol. 30, No.1, January, 2016 
$\wedge 9$

جدول رقم(9):نتائج تحليل التباين للفرق بين الأسعار المزرعية المقدرة والفعلى لمحصول القمح

\begin{tabular}{|c|c|c|c|c|}
\hline \multicolumn{5}{|c|}{ ANOVA } \\
\hline \multirow[t]{2}{*}{ data } & & & & \\
\hline & $\begin{array}{l}\text { Sum of } \\
\text { Squares }\end{array}$ & df & $\begin{array}{l}\text { Mean } \\
\text { Square }\end{array}$ & $F$ \\
\hline Between Groups & 12366140 & 7 & 1766591,4 & 5,73 \\
\hline Within Groups & 34498954 & 112 & 308026,38 & \\
\hline Total & 46865094 & 119 & & \\
\hline
\end{tabular}

المصدر: حسبت من بيانات الجدول رقم (^)،

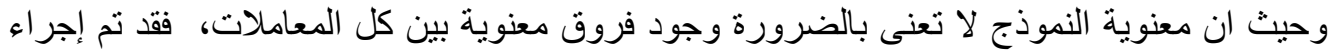

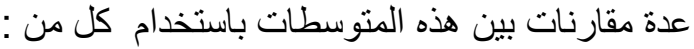

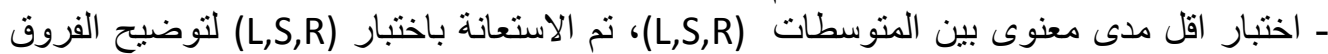

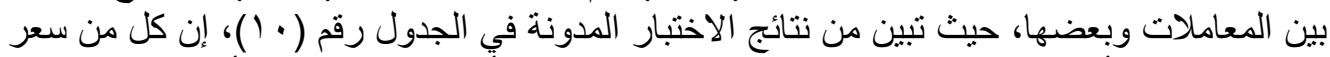

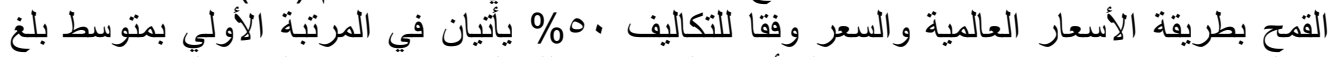

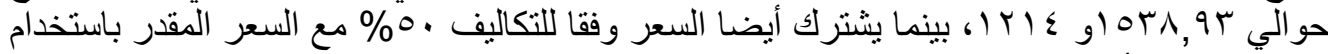

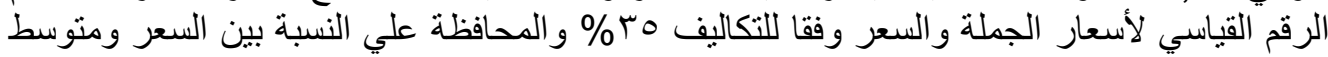

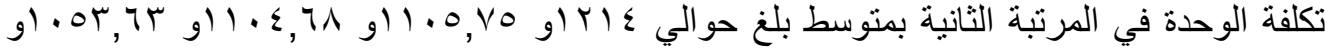

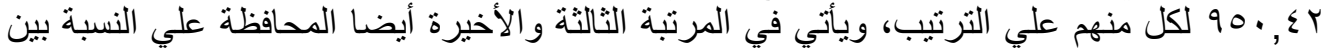

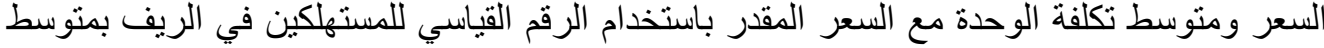

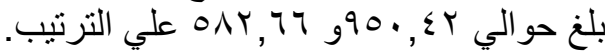

جدول رقم(· (1): نتائج اختبار اقل مدى معنوى (L,S,R) لمتوسطات الأسعار لمحصول القـح Duncan $(L, S, R)$

\begin{tabular}{|c|c|c|c|c|c|}
\hline \multicolumn{6}{|c|}{ Duncan $(L, S, R)$} \\
\hline \multicolumn{3}{|c|}{ alpha $=0,05$ Subset for } & \multirow{3}{*}{$\mathbf{N}$} & \multirow{3}{*}{ البديل } & factor \\
\hline \multirow{2}{*}{\multicolumn{3}{|c|}{ الترتيب }} & & & \multirow{2}{*}{ البدائل المقترحة } \\
\hline & & & & & \\
\hline & & $0 \wedge r, \pi T$ & 10 & 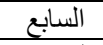 & السعر المقلار باستخذام الرقم القياسي للمستهلكين في الريف \\
\hline & $90 \cdot, \varepsilon r$ & $90 \cdot \leqslant \mathrm{T}$ & 10 & الخامس & المحافظة علي النسبة بين السعر ومتوسط تكلفة الؤوحدة \\
\hline & $1.0 \%, \pi$ & & 10 & الثالث & السعر وفقا لتكاليف الإتتاج المعدلة بro" \\
\hline & $11 \cdot \varepsilon, 7 \lambda$ & & 10 & الثانى & السعر وفقا لتكاليف الإنتاج الفعلية \\
\hline & $11.0,10$ & & 10 & 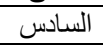 & السعر المقار باستخذام الرقم الققياسي لأسعار الجملة \\
\hline$|r| \leqslant, \cdots$ & $|r| \leqslant, \cdots$ & & 10 & الرابع & السعر وفقا لتكاليف الإتتاج المعدلّة . ه\% \\
\hline $104 \lambda, 94$ & & & 10 & الثامن & السعر للقمح بطريقة الأسعار العالمية \\
\hline & & & 10 & الأول & السعر المزرعي الفعلي \\
\hline$\cdot, \cdot \mathrm{V}$ & $\cdot$, ro $^{\circ}$ & $\cdot, \cdot \mathrm{V}$ & & & Sig. \\
\hline
\end{tabular}

المصدر: حسبت من بيانات الجدول رقم (^)،

Fayoum J. Agric. Res. \& Dev., Vol. 30, No.1, January, 2016 
9.

ـ اختبار Dunnett t (2-sided للمقارنة بين معنوية الفرق بين متوسط السعر الفعلى ومتوسطات

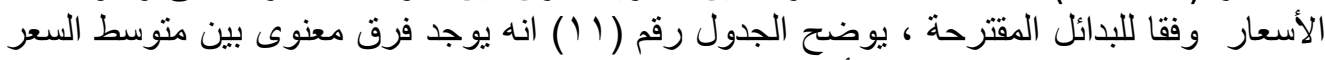

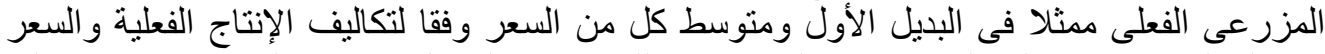

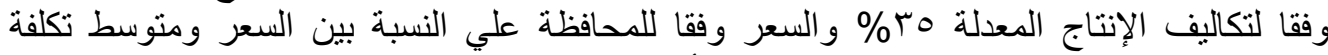
الوحدة و السعر المقدر باستخدام الرقم القياسي لأسعار الجملة والسعر المقدر باستخدام الرقم القياسي لإسي

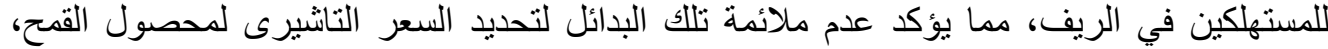

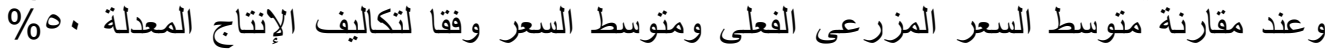

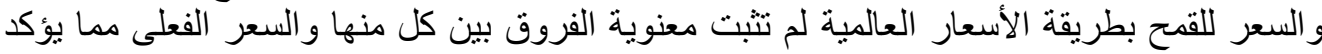

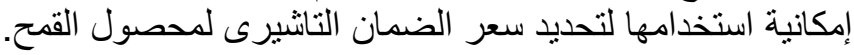

جدول رقم(1 (1): نتائج اختبار Dunnett t (2-sided) للمقارنة بين معنوية الفرق بين السعر الفعلى والأسعار وفقا للبدائل المقترحة

\begin{tabular}{|c|c|c|c|c|c|c|}
\hline \multicolumn{7}{|c|}{ Multiple Comparisons } \\
\hline \multicolumn{7}{|c|}{ Dunnett t (2-sided) } \\
\hline \multicolumn{2}{|c|}{$\begin{array}{l}\text { 95\% Confidence } \\
\text { Interval }\end{array}$} & \multirow{2}{*}{ Sig, } & \multirow{2}{*}{$\begin{array}{l}\text { Std, } \\
\text { Error }\end{array}$} & \multirow{2}{*}{$\begin{array}{c}\text { والمرق بين الفعلى (J-Jean (المقر Meance } \\
\text { Difference }\end{array}$} & \multirow{2}{*}{\multicolumn{2}{|c|}{ البدائل السعرية }} \\
\hline $\begin{array}{l}\text { Upper } \\
\text { Bound }\end{array}$ & $\begin{array}{l}\text { Lower } \\
\text { Bound }\end{array}$ & & & & & \\
\hline $12,17_{-}$ & $1.94,90_{-}$ & $\cdot, \cdot \varepsilon$ & $T \cdot Y, T T$ & $000, \cdot 0_{-}$ & الثانى & السعر وفقا لتكاليف الإنتاج الفعلية \\
\hline$T \Lambda, Y \cdot-$ & $11 \leqslant \varepsilon, \cdots$ & $\cdot, \cdot r$ & $r \cdot r, T T$ & $7 \cdot 7,1 \cdot-$ & الثالث & السعر وفقا لتكاليف الإنتاج المعدلة \%ro \\
\hline $94,1 V$ & $9 \wedge r, 7 r-$ & $\cdot, 10$ & $Y \cdot Y, T T$ & $\varepsilon \leqslant 0, V r_{-}$ & 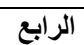 & السعر وفقا لتكاليف الإنتاج المعدلة . \%\% \\
\hline$|V|, \leqslant Y-$ & $|r \leq V, r|-$ & $\cdot, \cdots$ & $r \cdot r, T \tau$ & $V \cdot q, r_{-}$ & 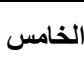 & تكلفة الوحافة علي النسبة بين السعر ومتوسط \\
\hline 17,99 & $1.91, \mathrm{~N}_{-}$ & $\cdot, \cdot \varepsilon$ & $r \cdot r, T \tau$ & oor, 99. & 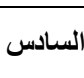 & لأسعار الجملة باستخدام الرقم القياسي \\
\hline orq, $1 V_{-}$ & $171 \leqslant, 9 V_{-}$ & $\cdot, \cdots$ & $r \cdot r, 7 \tau$ & $1 \cdot V v_{,} \cdot v_{-}$ & 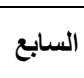 & للمستهر المقين في الريفذام الرقم القياسي \\
\hline$\sum 09, Y \mu$ & 717,07 & $1, \cdots$ & $Y \cdot Y, T T$ & $V \wedge, T V_{-}$ & 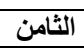 & السعر للقمح بطريقة الأسعار العالمية \\
\hline
\end{tabular}

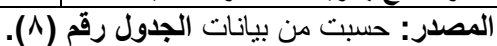

التوصيات: فى ضوء ما ورد بالبحث من نتائج توصى الدراسة :

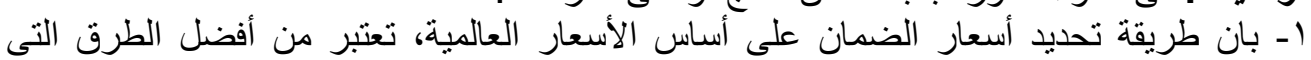
درست فى تحديد السعر المزرعى لمحصول القمح تلتها طريقة تحديد السعر وفقا لتكاليف الإنتاج المعدلة ، ٪\%، لذا توصى الدراسة باستخدام أكثر من طريقة فى ان واحد عند قيام الحكومة

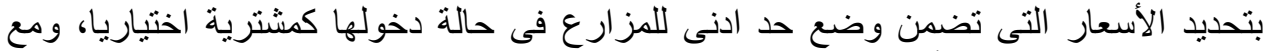

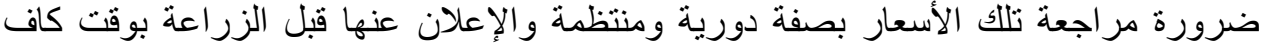

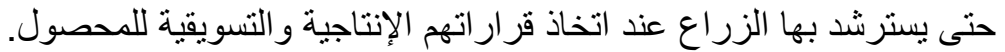

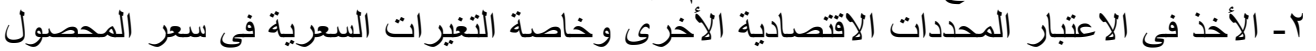

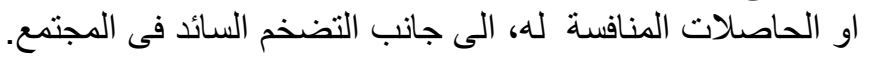
(الملخص:

تمنلت مشكلة الدراسة فى قلة الحوافز الكمية والنوعية الممنوحة لمنتجى القمح فى مصر كمحصول

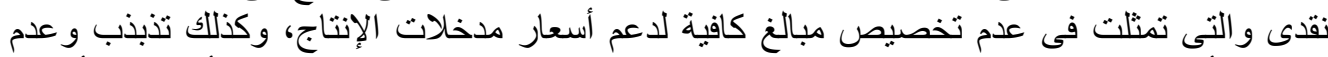

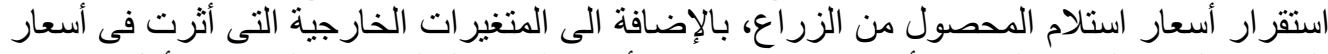

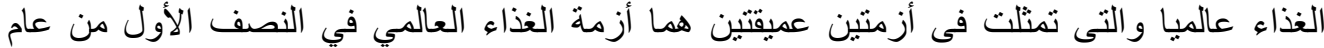

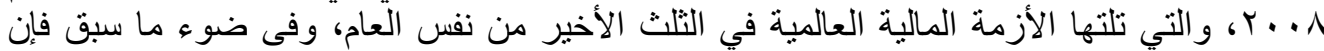

Fayoum J. Agric. Res. \& Dev., Vol. 30, No.1, January, 2016 
91

الهدف الرئيسي لهذا البحث هو التعرف على أهم المتغيرات الاقتصادية المؤثرة في جانبى الإنتاج

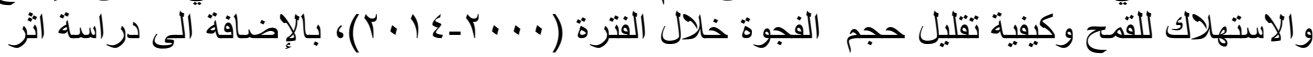

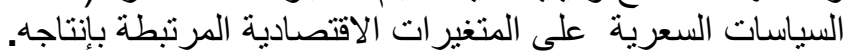

وقد أوضحث نتائج الاراسية:

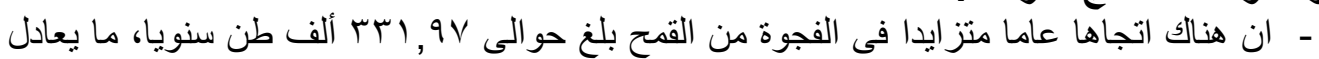

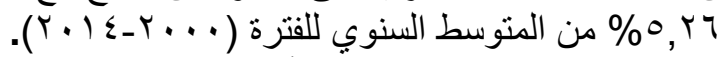

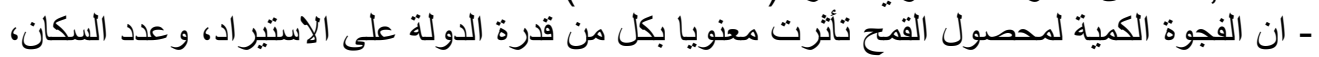

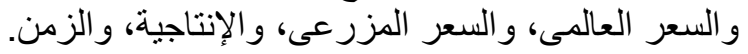

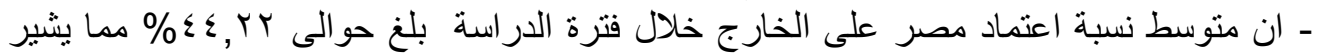
الى أن مصر تقع فى منطقة التبعية الغذائية الخطرة في محصول القمح حيث زاد اد معدل التبعية

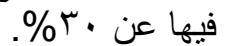

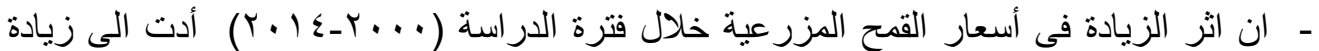

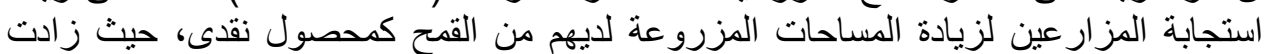

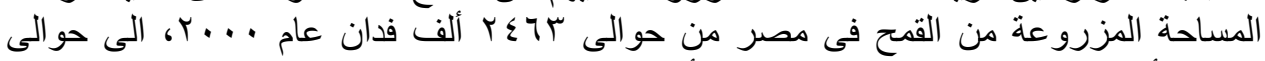

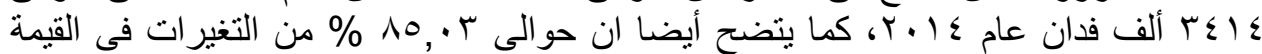

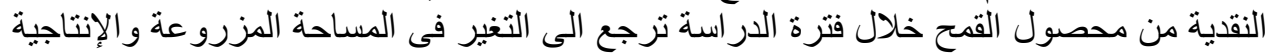

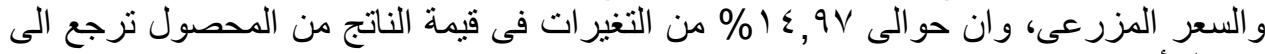
عو امل أخرى. - طريقة تحديد أسعار الضمان على أساس الأسعار العالمية، تعتبر من أفضل الطرق التى درست فى إلى تحديد السعر المزرعى لمحصول القمح تلتها طريقة تحديد السعر وفقا لتكاليف الإنتاج المعدلة

ا ـ الجهاز المركزي للتعبئة العامة والإحصاء، الكتاب الإحصاء السنوي، أعداد متفرقة.

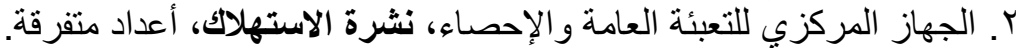

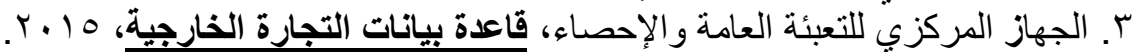

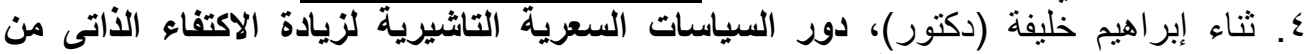

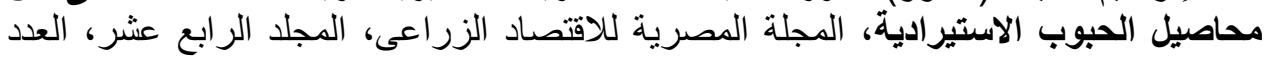

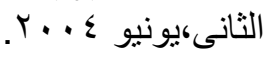
○. محمد حسن عيسى، سياسة التسعير فى القطاع الزراعى المصرى ودورها فى تحقيق الاكتفاء

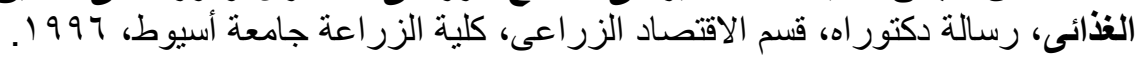

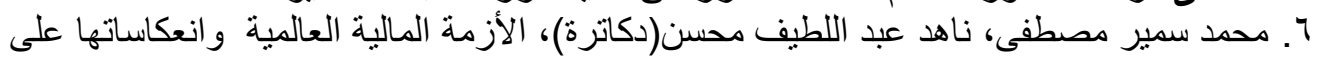

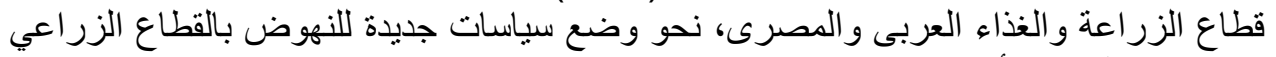

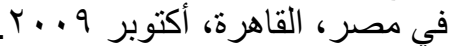
V. محمد صلاح الدين الجندى(دكتور)، الأزمة المالية العالمية تثخيصها، أسبابها، أثنارها،

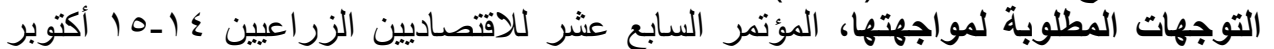
r... 9 ^. مصطفى محمد السعدنى ـألفت على ملوك(دكاترة)، الفجوة الغذائية بالوطن العربى،مجلة العلوم

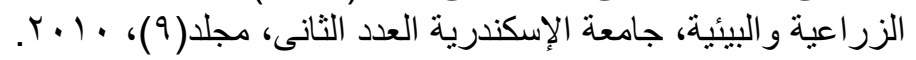

Fayoum J. Agric. Res. \& Dev., Vol. 30, No.1, January, 2016 
9. وزارة الزراعة، واستصلاح الأراضى، الإدارة المركزية للاقتصاد الزراعي، نثرة الاقتصاد الز الزراعي، أعداد متفرقة.

10- Ibrahim Soliman, Jacinto F, Fabiosa, Mohamed Gaber Amer, and Siham

Kandil, Impacts of the Economic Reform Program on the Performance of the Egyptian Agricultural Sector, 2010, Working Paper 10-WP 509, Center for Agricultural and Rural Development, Iowa State University, Ames, Iowa 50011-1070,

\title{
THE REALITY OF WHEAT PRODUCTION IN EGYPT UNDER GLOBAL ECONOMIC CHANGES AND THE POLICIES OF INDICATIVE PRICES
}

Yaser Abdel-Hamed Diab

Department of Agricultural Economics, Faculty of Agriculture, Assiut University, Assiut,

\begin{abstract}
The study problem can be presented as the lack of quantitative and qualitative motivation to producers of wheat in Egypt which can be expressed in the failure to allocate sufficient funds to support the prices of inputs and price fluctuation, Moreover, external variables, in the first half of 2008, affected global food prices, Therefore, the main objective of this research is to identify the most important economic variables affecting both sides of the production and consumption of wheat and how to reduce the size of the gap during the period (2000-2014), In addition to examining the impact of the policies on economic variables associated with its production.
\end{abstract}

The results of the study showed:

-There is a general trend of increasing in the gap of wheat; it was around 331,97 thousand tons per year, the equivalent of 5,26\% of the annual average.

-The quantity wheat gap affected morally by each State's ability to import, and population, and the world price, and farm price, productivity, and time.

-The average proportion of dependence on the abroad during the study period was about 44,22\% indicating that Egypt is in a hazardous food dependency where dependency rate protect about $30 \%$.

-The impact of increasing the farm price of wheat during the study period (2000-2014) led to increased turnout of farmers increase the areas planted with wheat as a cash crop.

- It is also clear that about $85,03 \%$ of changes in monetary value of wheat during the study period due to the change in area harvested, productivity and farm price, and about $14,97 \%$ from changes in the value of crop output due to other factors.

- Method of setting prices for wheat crop insurance on the basis of world prices considered as the best one between the other methods studied, followed

Fayoum J. Agric. Res. \& Dev., Vol. 30, No.1, January, 2016 
by determination of the price according to production costs of the crop with the average $50 \%$.

Fayoum J. Agric. Res. \& Dev., Vol. 30, No.1, January, 2016 